\title{
Miniaturized Charpy Specimens for the Indirect Verification of Small-Scale Charpy Machines: Initial Qualification Phase
}

Enrico Lucon

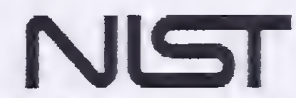




\title{
Miniaturized Charpy Specimens for the Indirect Verification of Small-Scale Charpy Machines: Initial Qualification Phase
}

\author{
Enrico Lucon \\ Materials Reliability Division \\ National Institute of Standards and Technology \\ 325 Broadway \\ Boulder, CO 80305
}

March 2012

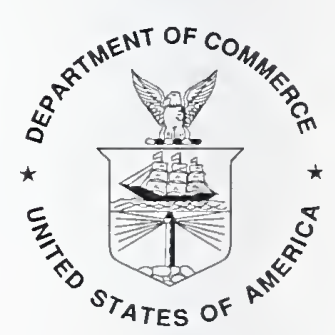

U.S. Department of Commerce John E. Bryson, Secretary

National Institute of Standards and Technology Patrick D. Gallagher, Under Secretary of Commerce for Standards and Technology and Director 
Certain commercial entities, equipment, or materials may be identified in this document in order to describe an experimental procedure or concept adequately. Such identification is not intended to imply recommendation or endorsement by the National Institute of Standards and Technology, nor is it intended to imply that the entities, materials, or equipment are necessarily the best available for the purpose.

National Institute of Standards and Technology Technical Note 1562

Natl. Inst. Stand. Technol. Tech. Note 1562, 24 Pages (March 2012)

CODEN: NTNOEF 


\section{Abstract}

Small specimen test teehniques beeome ever more popular as the need increascs to eharaeterize mechanieal propertics by use of the smallest possible amount of material, duc to various restrietions on material availability, irradiation, testing space, and other faetors.

NIST is eurrently developing referenee miniaturized Charpy V-noteh (MCVN) specimens for the indirect verifieation of small-seale impaet testing maehines. The same materials used for NIST standard verification specimens arc bcing evaluated at three cnergy lcvels (low, high and super-high). Two speeimen types are being investigated, denominated KLST (from the German Kleinstprobe, or "small speeimen") and RHS (redueed half-size).

Scveral instrumented impaet tests on miniaturized KLST and RHS specimens of low, high and super-high encrgy have been performed and analyzed. The variability of MCVN data has been compared to that of full-size Charpy data from the same lot of test speeimens.

Although this ean be eonsidered just the preliminary phase of this projeet, the results indicate that MCVN verifieation speeimens ean be used for the indireet verification of small-scale instrumented impact testers, both in terms of absorbed energy and maximum foree.

Additional aspeets have also been investigated, sueh as the influenee of shear lip symmetry and speeimen fraeture on absorbed energy and the eorrelation between miniaturized and full-size Charpy data.

\section{Keywords}

Indireet verifieation; KLST; MCVN; miniaturized Charpy speeimens; RHS; small speeimens; small-scale impaet testers. 


\section{$\underline{\text { Table of Contents }}$}

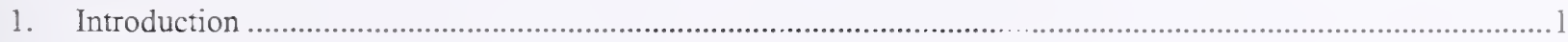

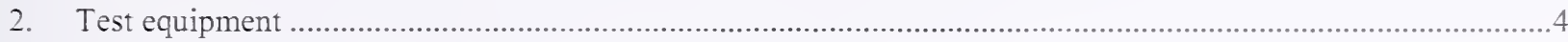

3. Preliminary qualification of NIST small-scale instrumented impact tester: tests on JRQ pressure vessel steel ....8

4. Development of MCVN SRM's: preliminary testing of low, high and super-high KLST and RHS specimens.12

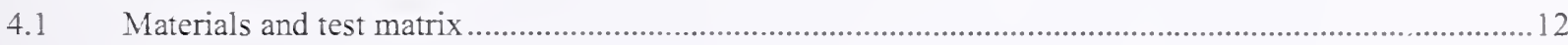

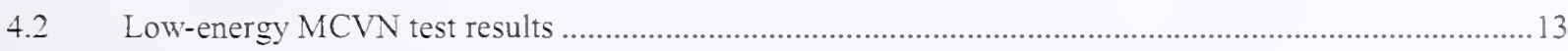

4.2.1 Calculation of sample size and outlier analysis ......................................................................14

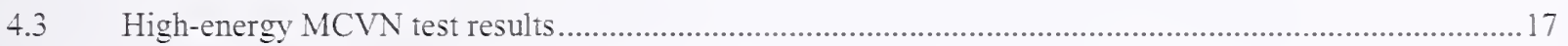

4.3.1 Calculation of sample size and outlier analysis .......................................................................... 18

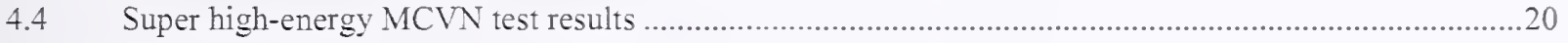

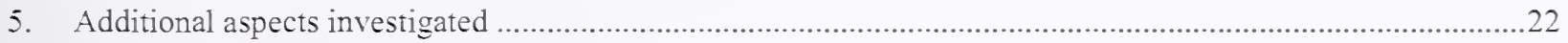

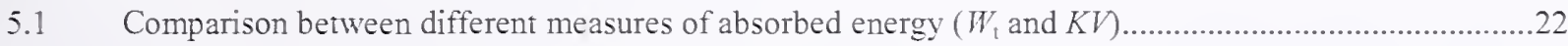

5.2 Correlation between full-size and miniaturized specimen absorbed energies ..........................................22

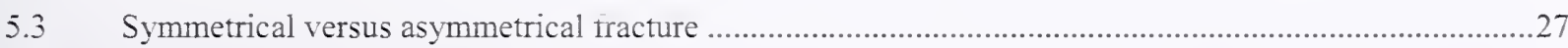

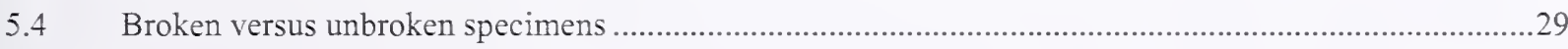

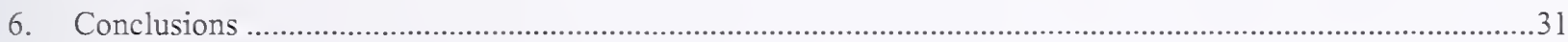

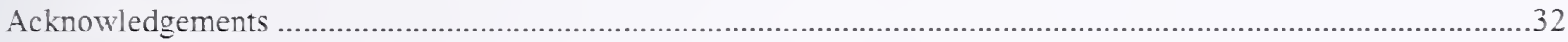

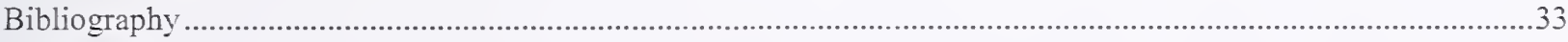




\section{Introduction}

Evaluating the mechanical properties of components or structures is typically a destructive approach, since it requires direct material sampling. This is often possible only if the sample size is so small that easy repairing, or even no repairing, is needed for further operation of the component. This is the case of hardncss mcasurements, which can be considered practically nondestructive. This report documents the initial (qualification) phase of a NIST project whose objective is to develop a new standard reference material (SRM): miniaturized Charpy specimens for the indirect vcrification of small-scale impact testing machines.

Impact test results are needed for the integrity analysis and residual life assessment of reactor pressure vessels, both for current (Generation II) and near-future (Generation III) nuclear power plants. For these reactors, radiation-induced degradation is monitored by means of a surveillance program, which includes testing of standard-size Charpy impact specimens that have been stored in capsules inside the rcactor vessels (surveillance capsules). Additional data can be gathered from these surveillance specimens by extracting miniaturized impact specimens from the broken, full-size Charpy specimens. For future advanced nuclear reactors (Gen IV, fusion, accelerator-driven systems), the main restriction is the limited available space for irradiating structural materials under relevant conditions. Miniaturization of the samples, including impact specimens, is therefore an efficient way to optimize both material consumption and irradiation space.

Laboratories testing standard Charpy specimens can indirectly verify their pendulum machines through the use of SRMs provided by national metrology laboratories such as NIST or IRMM (Institute for Reference Materials and Measurements). For laboratories testing miniaturized Charpy specimens with small-scale pendulums (i.e., reduced-scale impact machines with potential energy between $15 \mathrm{~J}$ and $50 \mathrm{~J}$ and impact speed around 3.8 $\mathrm{m} / \mathrm{s}$ ), no verification specimens are currently available to check the state of calibration of their machines. Past experience with full-sizc impact machines indicates that without indirect verification, the reliability of the test data and their value for comparison with data from other laboratories is significantly reduced [1-3].

Historically, miniaturized Charpy V-notch (MCVN) specimens have been used since the 1980s in many countries, mainly as a means of re-using already-tested Charpy samples. The non-proportional specimens typically used in Europe are designated KLST (from the German Kleinstprobe, or "small specimen") or ESIS (from the acronym of the European Structural Integrity Society) specimens, and have the following nominal dimensions: thickness, $3 \mathrm{~mm}$; width, $4 \mathrm{~mm}$; length, $27 \mathrm{~mm}$; notch depth, $1 \mathrm{~mm}$ (i.e., square cross section under the notch); see also Figure 1. Extensive data sets have been generated with this specimen, particularly on nuclear pressure vessel steels [4].

The KLST specimen was the first MCVN type to be included in an international test standard, when in 2006 an amendment (Annex D) titled "Instrumented Charpy V-notch pendulum impact test of sub-size test pieccs" was approved for inclusion in the ISO 14556:2000 standard [5].
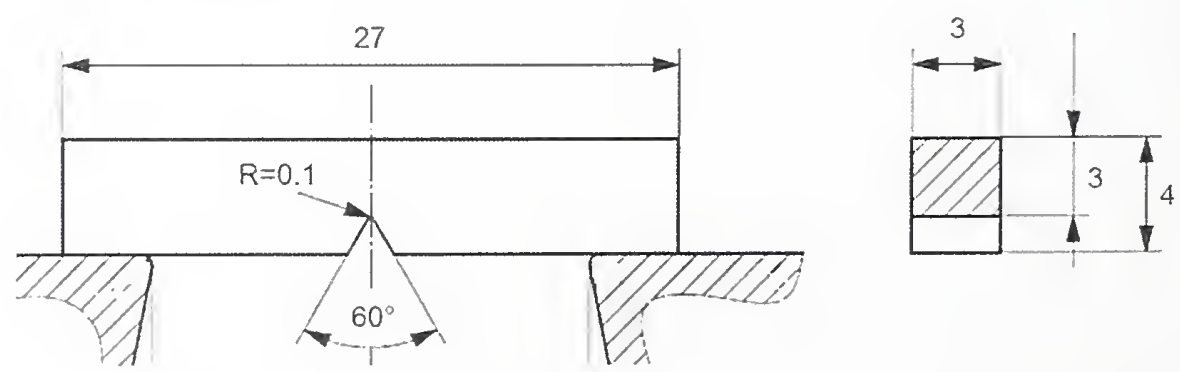

Figure 1 - KLST-type MCVN specimen (dimensions in millimeters). 
The proportional (scaled) MCVN spccimen preferred in the United States is either the "half-sizc" (HS) specimen (dimensions $5 \mathrm{~mm} \times 5 \mathrm{~mm} \times 27.5 \mathrm{~mm}$ ) or the "reduced half-size" (RHS) specimen $(4.83 \mathrm{~mm} \times 4.83 \mathrm{~mm}$ $\times 24.13 \mathrm{~mm}-$ see Figure 2). The RHS configuration obviously derives from the HS geometry and aims at optimizing the extraction of four MCVN specimens from a broken full-size Charpy half with consideration for the material consumed by the cutting operations. RHS specirnens represent the reference MCVN geometry for the ASTM E2248 standard [7], which was officially issued in 2009.
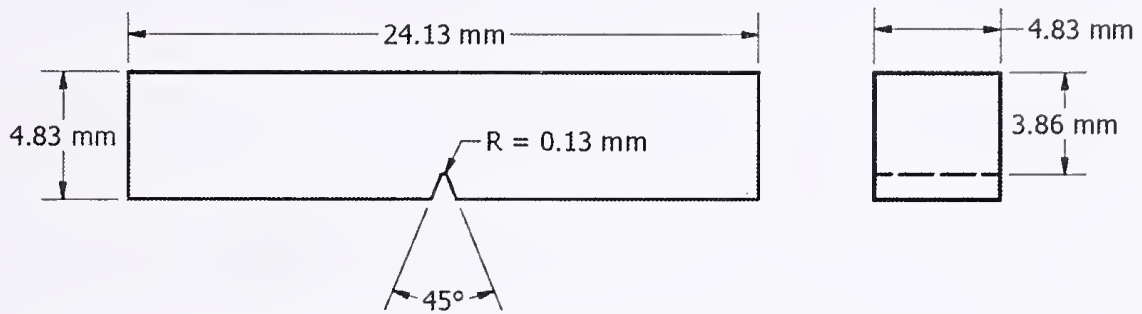

Figure 2 - RHS-type MCVN specimen.

It is important to stress the distinction between miniaturized and sub-size Charpy specimens. The latter are documented in Annex A3 (Additional Impact Test Specimen Configurations) of ASTM E23-07a ${ }^{\varepsilon 1}$. In these specimens, one of the cross-sectional dimensions (either width or thickness) retains the value of full-size samples $(10 \mathrm{~mm})$, whereas the other is reduced to $2.5 \mathrm{~mm}, 3 \mathrm{~mm}, 5 \mathrm{~mm}$, or $7.5 \mathrm{~mm}$. For the record, this Annex also includes the HS geometry ( $5 \mathrm{~mm} \times 5 \mathrm{~mm}$ cross-section) and an additional $5 \mathrm{~mm} \times 20 \mathrm{~mm}$ configuration. Conversely, the MCVN specimens used in this investigation have all linear dimensions reduced with respect to those of a full-size Charpy V-notch (CVN) specimen.

The advanced reactor community, i.e., laboratories working for Generation IV [8] and fusion nuclear reactors, typically uses MCVN specimens for characterizing and qualifying candidate structural materials. In particular, the International Fusion Materials Irradiation Facility (IFMIF) [9], which is expected to closely simulate the irradiation conditions of a fusion power reactor, will have a limited irradiation volume and will therefore rely on the use of very small samples including MCVN specimens. For these advanced reactor applications, codes and regulations have not been developed yet. It is, however, almost certain that small-scale and miniaturized specimens will play a leading role in future regulations, and it would therefore be beneficial to have MCVN SRMs available for consideration as regulations are developed.

Another technical community that would potentially benefit from a more widespread use and public acceptance of MCVN testing is the pipeline community. When product-form dimensions do not allow extraction of full-size Charpy specimens, as in the case of thin-walled pipes or tubes, specimens with reduced size have to be used.

MVCN specimens have also been tested [10] on a conventional, full-size impact tester, provided that anvils and supports are adequately modified to account for specimen span and dimensions. However, the recommended procedure is to use a small-scale pendulum that has a significantly lower potential energy (15 J to $50 \mathrm{~J}$ instead of $300 \mathrm{~J}$ or more) and slightly lower impact speed $(3.8 \mathrm{~m} / \mathrm{s}$ instead of $5 \mathrm{~m} / \mathrm{s}$ or more). Testing MCVN specimens on a full-size pendulum can be considered equivalent to using a load cell with $100 \mathrm{kN}$ capacity for testing sub-size tensile specimens that exhibit maximum forces in the order of a few kilonewtons.

Small-scale impact testers cannot be indirectly verified by means of standard Charpy reference specimens, for both dimensional and energy reasons. On the other hand, MCVN specimens with certified values of absorbed 
energy or maximum force are currently unavailable. Therefore, users of small-scale impact tester have no means of verifying the performance of their machine by means of an approach equivalent to ASTM E23 or ISO 148-2.

The current project aims at developing and qualifying MCVN reference specimens with certified absorbed cnergy and maximum force values for both KLST and RHS samples. These objectives will bc attained through the following steps:

(a) Establishment of a general procedure for machining MCVN specimens from previously tested NIST reference samples (low, high and super-high energy levels), including dimensional requirements that are stricter than those currently enforced in the ASTM E2248-09 standard.

(b) Initial qualification of the MCVN low, high and super-high reference specimens by performing a statistically relevant number of instrumented tests at NIST with a small-scale pendulum, which has been purchased for this investigation.

(c) Statistical evaluation of the results through the use of qualification procedures similar to those used for CVN samples [3], in order to confirm that MCVN specimens satisfy the requirements for standard reference matcrials.

(d) Final qualification of the MCVN reference specimens through the organization, coordination and execution of an interlaboratory exercise (Round-Robin) in accordance with ASTM E691-09 [11], involving US and international laboratories.

(e) Statistical evaluation of the Round-Robin results according to ASTM E691-09 [11] and NIST qualification procedures [3].

(f) Establishment of the MCVN indirect verification procedure and the corresponding requirements that users will have to comply with in order to verify their small-scale machine, to be eventually adopted by both ASTM E2248 and ISO 14556 standards.

Additional objectives are:

- The development of data base to demonstrate the usefulness of MCVN specimens for typical pipeline stcels.

- Promoting the use of MCVN reference specimens within the communities that represent the most suitable targets (advanced nuclear reactors and pipelines).

Finally, it is worth noting that the feasibility of fabricating MCVN verification specimens of the same materials and energy levels used for existing CVN reference specimens was demonstrated in a collaboration between IRMM (EU reference specimen producer) and SCK • CEN (Belgian Nuclear Center) [12]. Those results justify our decision to use the same materials employed by the Charpy program at NIST for full-size verification specimens. 


\section{Test equipment}

A small-scale impact tester (table-top pendulum machine), equipped with instrumented strikers for testing KLST and RHS specimens, was purchased by the NIST Materials Reliability Division for use in this project.

The parts that need to be changed when testing either MCVN type (KLST or RHS) are the hammer, which includes the instrumented striker, and the supports/anvils block. Weight plates mounted on the hammer are provided to obtain the required potential eriergy.

The impact energy capacity of the machine, corresponding to a fall angle of $160^{\circ}$, is $50.1 \mathrm{~J}$ for KLST and 50.8 for RHS. The nominal impact velocity is $3.5 \mathrm{~m} / \mathrm{s}$ for both configurations. KLST specimens were tested with a $2 \mathrm{~mm}$ striker conforming to ISO 148-2 (Figure 3), while for RHS specimens a scaled-down $8 \mathrm{~mm}$-striker was used in accordance with ASTM E2248 (reduced 4 mm striker, Figure 4).

The nominal distance between specimen supports (span) is $22 \mathrm{~mm}$ for KLST and $19.3 \mathrm{~mm}$ for RHS.



Figure 3 - Dimensions of the KLST striker.



Figure 4 - Dimensions of the RHS striker. The striker width is $8 \mathrm{~mm}$. 
The NIST small-scale table-top impact tester is shown in Figure 5 (general view), Figure 6 (detailed view of the hammer and the instrumented striker) and Figure 7 (detailed view of the specimen supports and anvils).

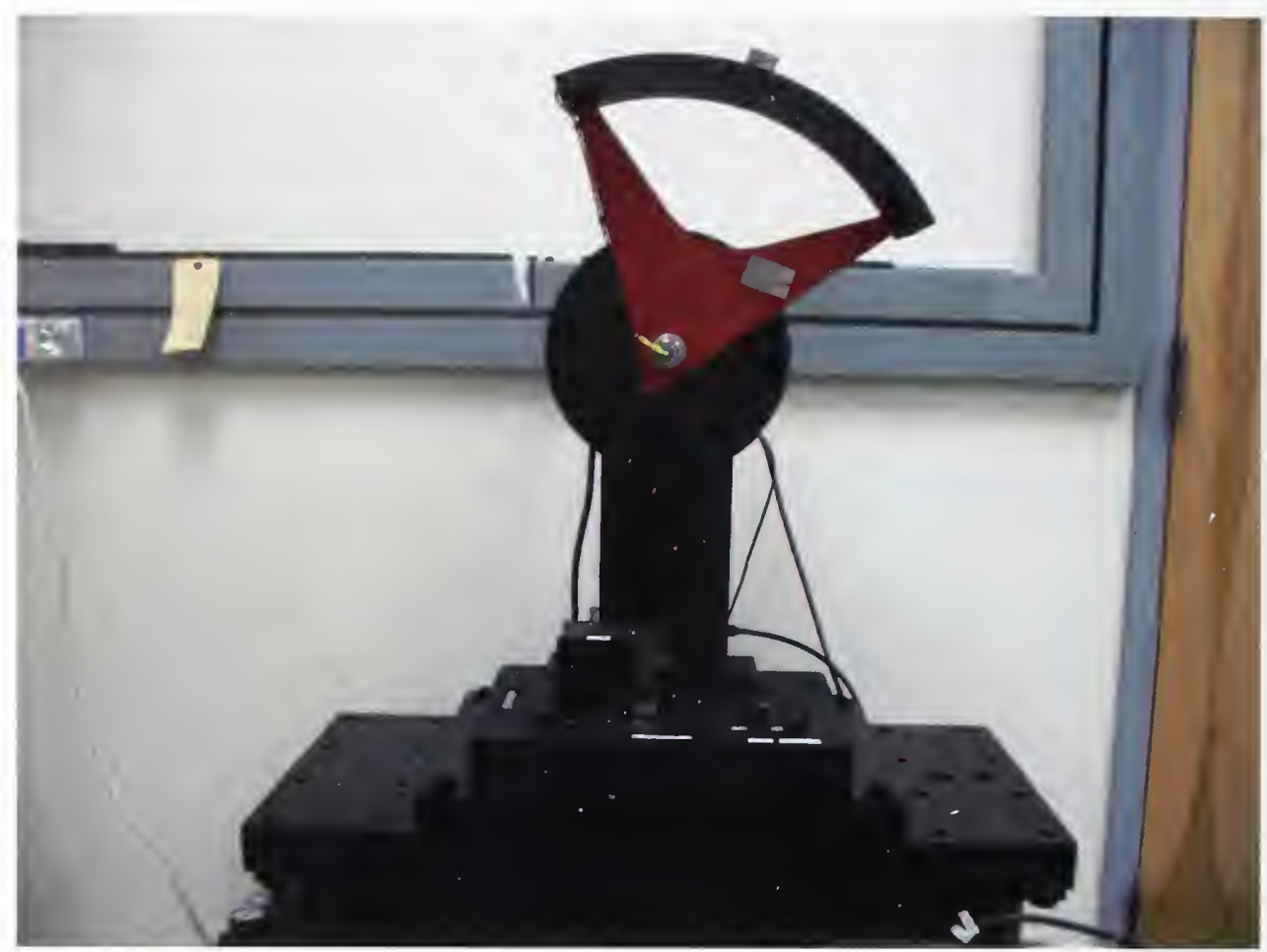

Figure 5 - General view of the NIST small-scale table-top instrumented impact tester.



Figure 6 - Detailed view of the hammer and instrumented striker of the NIST small-scale table-top instrumented impact tester. 




Figure 7 - Detailed view of the supports and anvils of the NIST small-scale table-top instrumented impact tester with a KLST specimen in test position.

The energy absorbed by specimen fracture is calculated from the difference between the fall angle (in the latched position) and the rise angle of the hammer. The angular position of the hammer is monitored by an optical encoder that has an energy resolution of $0.006 \mathrm{~J}$. Values of absorbed energy are corrected for windage and friction; the correction is approximately $0.2 \mathrm{~J}$ for both configurations.

Both instrumented strikers were statically calibrated by the manufacturer in accordance with E2248-09 and ISO 14556:2000. The striker voltage/applied force calibration data for both strikers are shown in Figure 8. The KLST striker was calibrated up to an applied compressive force of $3.11 \mathrm{kN}(700 \mathrm{lb})$, while the RHS striker was calibrated up to $10 \mathrm{kN}(2250 \mathrm{lb})$. Both strikers exhibited a fully linear behavior.

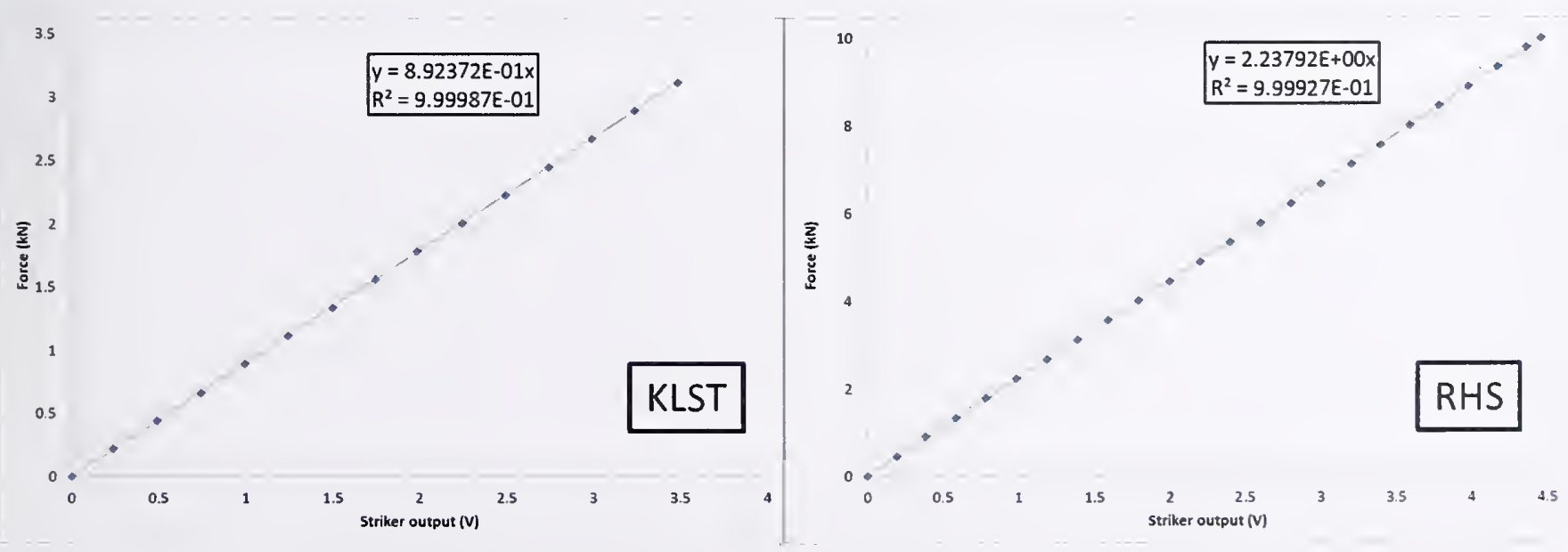

Figure 8 - Static calibration data and linear correlation trend lines for the KLST (left) and RHS (right) instrumented strikers. 
The signal of the strain gages from the instrumented strikers is fed through a conditioner stage that amplifies it by a factor 300 . The striker signal is then channeled through an analog/digital acquisition unit, which is connected by a USB cable to a computer for time/force data storage and analysis. The sampling rate is $1 \mathrm{MHz}$. The optical encoder is directly connected to the computer, where the test software converts the readings into values of absorbed energy. 


\section{Preliminary qualification of NIST small-scale instrumented impact tester: tests on JRQ pressure vessel steel}

Before testing MCVN specimens extracted from NIST verification specimens. we performed a preliminary qualification of our test equipment by running KLST instrumented tests on a steel that was used by the ESIS TC5 Working Group for a Round-Robin exercise in the mid-90s [13].

The steel, labeled JRQ, is a reactor pressure vessel steel of the ASTM A533B Cl.1 type, used by the International Atomic Energy Authority (IAEA) as a correlation monitor material in several studies of irradiation-induced material embrittlement [14]. The ESIS TC5 Round-Robin involved 14 international laboratories, who performed instrumented impact tests on KLST specimens of JRQ steel on a number of different tests machines including full-scale and small-scale pendulums, a small-scale drop tower and a high-rate universal testing machine. Phase 1 of the Round-Robin consisted of room temperature tests, whereas in Phase 2 participants performed tests at different temperatures spanning linear elastic, elastic-plastic and ductile-to-brittle transitional behavior for the investigated steel [13]. All the KLST specimens used in the Round-Robin were manufactured from a block designated "ESIS" and distributed to participants by SCK •CEN, the Belgian Nuclear Center.

Thirteen KLST specimens of JRQ steel, from the same lot that had been prepared for the ESIS TC5 Round-Robin, were shipped from SCK •CEN to NIST at the end of 2011. Eight of these were tested at room temperature at NIST, six instrumented and two non-instrumented: the remaining five specimens are kept in storage for future testing.

For every instrumented test performed. values of force $F$, displacement $s$ and absorbed energy $W$ were determined in accordance with ASTM E2298-09 [15] and ISO 14556:2000 [5] at general yield (subscript gi), maximum force (subscript $m$ ), and test termination (subscript $t$ ). For both non-instrumented and instrumented tests. values of absorbed energy as measured by the optical encoder, $K V$, were also recorded. Test results are shown in Table 1, which also reports the ratio between absorbed energies provided by the encoder $(K I)$ and calculated from the area under the force/displacement test record, $W_{i}$, as well as the absolute difference between $W_{i}$ and $K V$.

Examination of Table 1 reveals that the standard deviation of force values is much lower than that for absorbed energy. The relative scatter of displacement values is intermediate between those of force and energy. except for values at general yield. The ratio $K V / W$ between instrumented and encoder energy is extremely: consistent, and the absolute difference between $K V$ and $W_{t}$ ranges between $0.7 \mathrm{~J}$ and $0.8 \mathrm{~J}$.

Table 1 - Results of KLST tests performed at NIST on JRQ specimens from the ESIS block. (") Yon-instrumented tests.

\begin{tabular}{|c|c|c|c|c|c|c|c|c|c|c|c|}
\hline $\begin{array}{l}\text { Specimen } \\
\text { id }\end{array}$ & $\begin{array}{c}T \\
\left({ }^{\circ} \mathrm{C}\right)\end{array}$ & $\begin{array}{c}F_{\mathrm{gv}} \\
(\mathrm{kN})\end{array}$ & $\begin{array}{c}F_{m} \\
(\mathrm{kN})\end{array}$ & $\begin{array}{c}s_{\underline{g v}} \\
(\mathrm{~mm})\end{array}$ & $\begin{array}{c}s_{m s} \\
(\mathrm{~mm})\end{array}$ & $\begin{array}{c}s_{I} \\
(\mathrm{~mm})\end{array}$ & $\begin{array}{l}W_{m s} \\
(J)\end{array}$ & $\begin{array}{l}\pi_{i}^{-} \\
(J)\end{array}$ & $\begin{array}{l}K I^{\prime} \\
(J)\end{array}$ & $K \vdash W_{i}$ & $\begin{array}{c}W_{r}-K V^{\top} \\
(\mathrm{J})\end{array}$ \\
\hline E519 & 21 & 1.01 & 1.33 & 0.21 & 2.18 & 11.48 & 2.56 & 8.08 & 7.37 & 0.912 & 0.71 \\
\hline E520 & 21 & 1.02 & 1.33 & 0.22 & 2.02 & 11.35 & 2.35 & 8.77 & 7.99 & 0.911 & 0.78 \\
\hline$E \Sigma+2$ & 21 & 0.99 & 1.32 & 0.28 & 2.14 & 11.45 & 2.38 & 8.69 & 7.92 & 0.911 & 0.77 \\
\hline$E 5+5$ & 21 & 1.01 & 1.33 & 0.20 & 2.07 & 11.02 & 2.42 & 8.49 & 7.72 & 0.909 & 0.77 \\
\hline E546 & 21 & 1.02 & 1.33 & 0.22 & 2.07 & 11.27 & 2.41 & 8.44 & 7.67 & 0.909 & 0.77 \\
\hline$E 547^{(*)}$ & 21 & - & - & - & - & - & - & - & 7.31 & - & - \\
\hline E548 (*) & 21 & - & . & - & - & - & - & - & 7.56 & - & - \\
\hline E549 & 21 & 1.01 & 1.29 & 0.25 & 2.04 & 11.19 & 2.29 & 8.79 & 8.03 & 0.914 & 0.76 \\
\hline \multicolumn{2}{|c|}{ Average } & 1.01 & 1.32 & 0.23 & 2.09 & 11.29 & 2.21 & 8.54 & 7.70 & 0.911 & 0.76 \\
\hline \multicolumn{2}{|c|}{ Standard deviation } & $\begin{array}{c}0.01 \\
1.08 \%\end{array}$ & $\begin{array}{c}0.02 \\
1.21 \%\end{array}$ & $\begin{array}{c}0.03 \\
12.90 \%\end{array}$ & $\begin{array}{c}0.06 \\
2.93 \%\end{array}$ & $\begin{array}{c}0.17 \\
1.53 \%\end{array}$ & $\begin{array}{c}0.09 \\
3.77 \%\end{array}$ & $\begin{array}{c}0.27 \\
3.15 \%\end{array}$ & $\begin{array}{c}0.27 \\
3.55 \%\end{array}$ & $\begin{array}{l}0.002 \\
0.19 \% \%\end{array}$ & $\begin{array}{c}0.03 \\
3.33 \%\end{array}$ \\
\hline
\end{tabular}


NIST data from Table 1 were compared with the results of Phase 1 of the ESIS TC5 Round-Robin (room temperature tests); see Table 2 and Figure 9 to Figure 12.

Table 2 - Comparison between ESIS Round-Robin and NIST results (average and standard deviation). $s_{r}$ is the repeatability standard deviation according to ASTM E691-11*.

\begin{tabular}{|c|c|c|c|c|c|c|c|c|c|}
\hline \multicolumn{2}{|c|}{ Data set } & $\begin{array}{c}F_{g y} \\
(\mathrm{kN})\end{array}$ & $\begin{array}{c}F_{m} \\
(\mathrm{kN})\end{array}$ & $\begin{array}{c}s_{g y} \\
(\mathrm{~mm})\end{array}$ & $\begin{array}{c}s_{m} \\
(\mathrm{~mm})\end{array}$ & $\begin{array}{c}s_{t} \\
(\mathrm{~mm})\end{array}$ & $\begin{array}{c}W_{m} \\
(\mathrm{~J})\end{array}$ & $\begin{array}{c}W_{t} \\
(\mathrm{~J})\end{array}$ & $\begin{array}{c}K V \\
(\mathrm{~J})\end{array}$ \\
\hline \multirow{2}{*}{$\begin{array}{c}\text { ESIS R-R } \\
{[13]}\end{array}$} & Average & 0.98 & 1.31 & 0.18 & 1.91 & 11.79 & 2.21 & 8.35 & 8.22 \\
\cline { 2 - 10 } & $s_{r}$ & 0.04 & 0.05 & 0.04 & 0.14 & 1.36 & 0.22 & 0.71 & 0.71 \\
\hline \multirow{3}{*}{ NIST } & Average & 1.01 & 1.32 & 0.23 & 2.09 & 11.29 & 2.40 & 8.54 & 7.70 \\
\cline { 2 - 10 } & $\begin{array}{c}\text { Standard } \\
\text { deviation }\end{array}$ & $\begin{array}{c}0.01 \\
1.08 \%\end{array}$ & $\begin{array}{c}0.02 \\
1.21 \%\end{array}$ & $\begin{array}{c}0.03 \\
12.90 \%\end{array}$ & $\begin{array}{c}0.06 \\
2.93 \%\end{array}$ & $\begin{array}{c}0.17 \\
1.53 \%\end{array}$ & $\begin{array}{c}0.09 \\
3.77 \%\end{array}$ & $\begin{array}{c}0.27 \\
3.15 \%\end{array}$ & $\begin{array}{c}0.27 \\
3.55 \%\end{array}$ \\
\hline
\end{tabular}

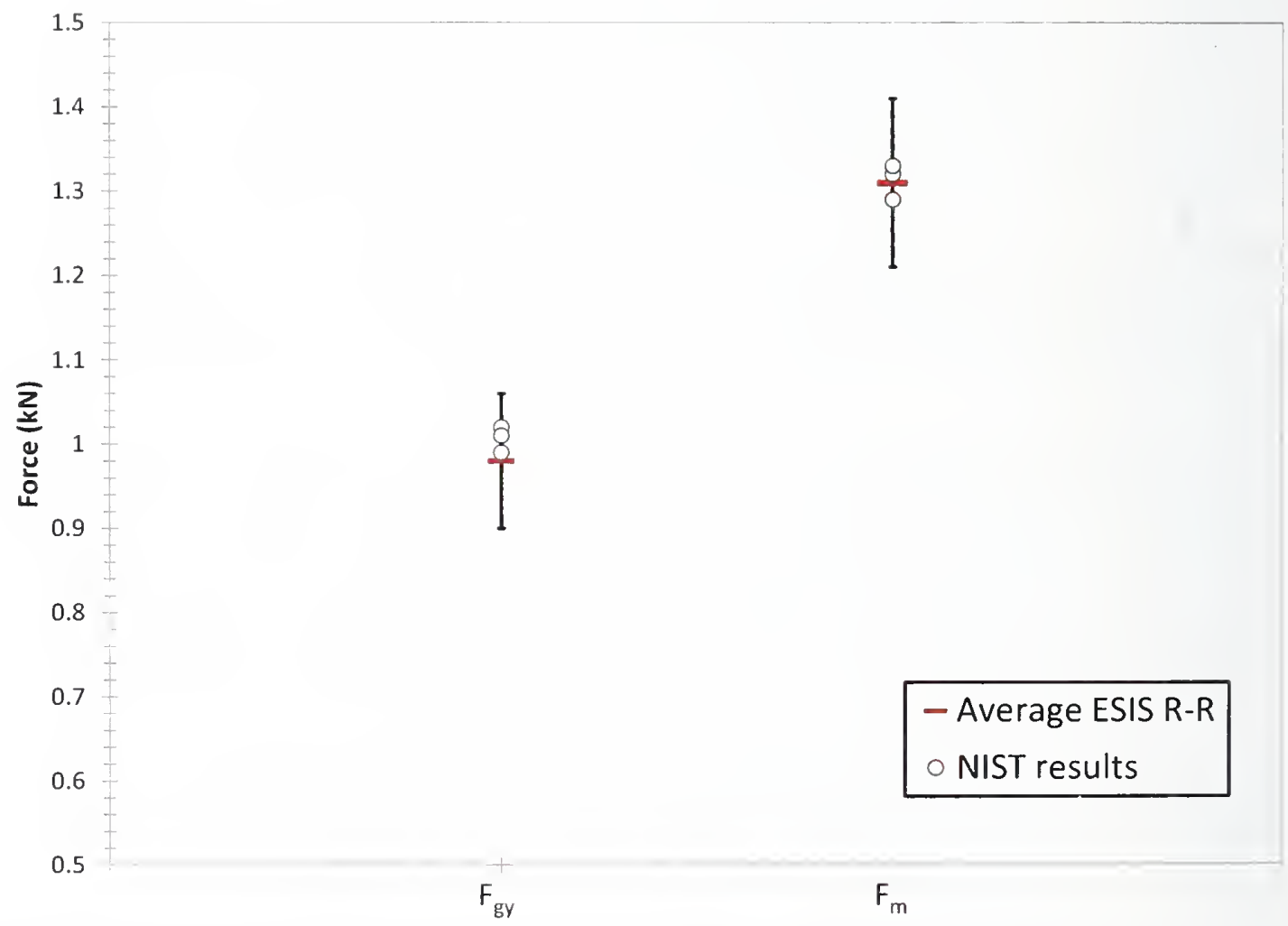

Figure 9 - Instrumented force values: comparison between ESIS Round-Robin and NIST results. The error bars are for the Round-Robin data and correspond to $\pm 2 s_{r}$.

*ASTM E691-11 definition of $s_{r}$ : standard deviation of test results obtained under repeatability conditions, i.e., conditions where independent test results are obtained with the same method on identical test items in the same laboratory by the same operator using the same equipment within short intervals of time. 


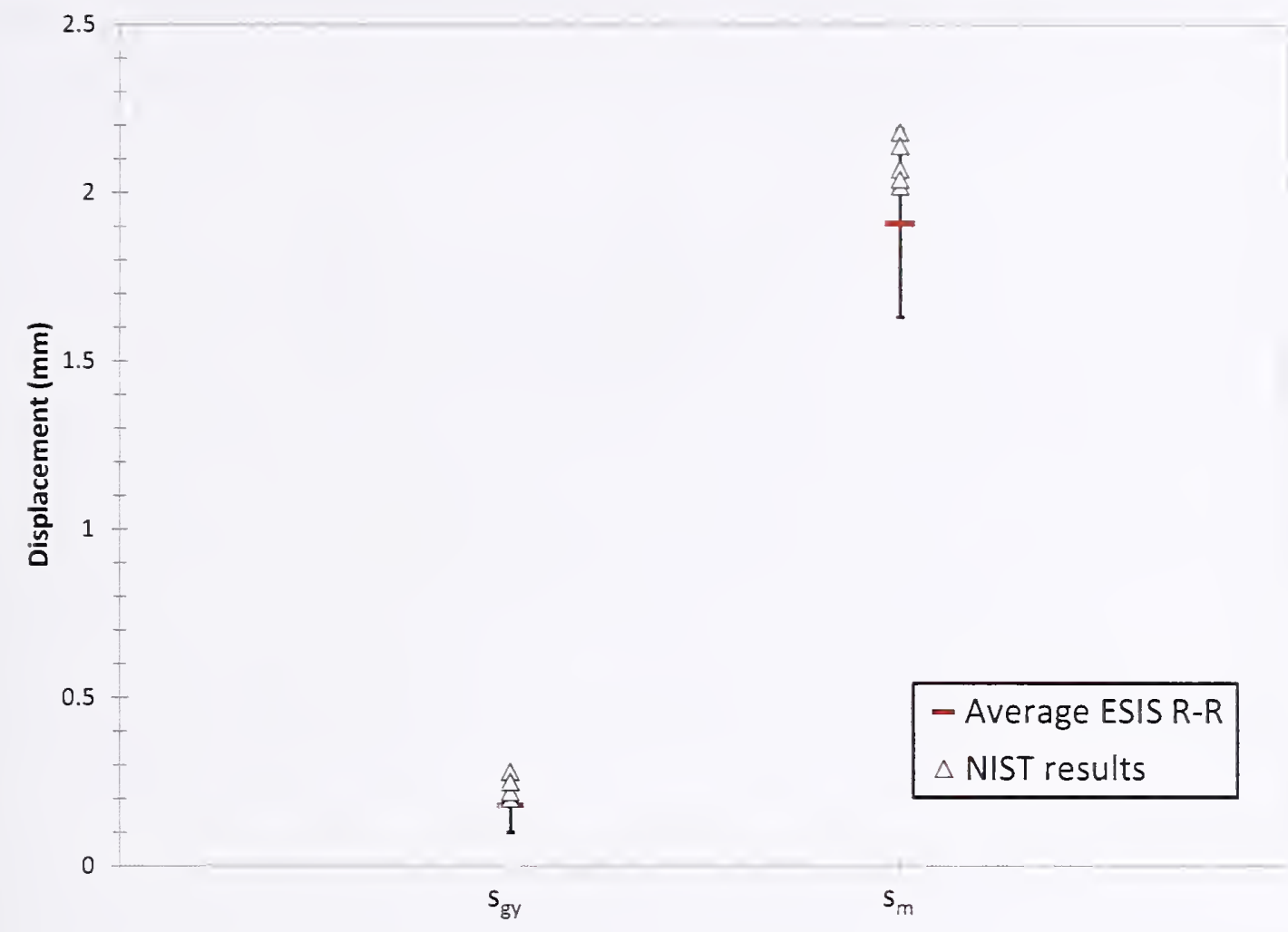

Figure 10 - Instrumented displacement values $s_{g y}$ and $s_{m}$ : comparison between ESIS Round-Robin and NIST results. The error bars are for the Round-Robin data and correspond to $\pm \mathbf{2} s_{r}$.

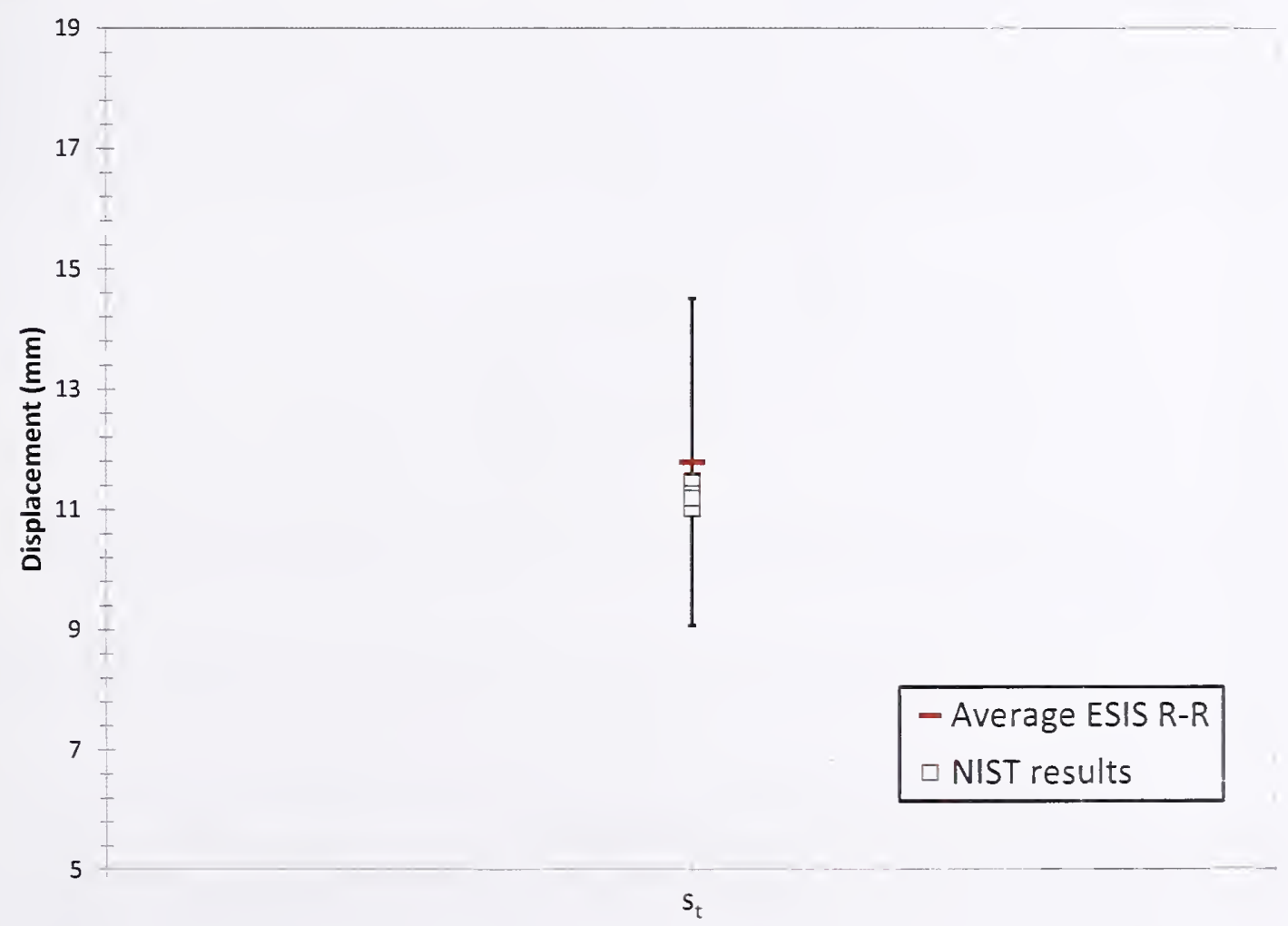

Figure 11 - Instrumented displacement values $s_{t}$ : comparison between ESIS Round-Robin and NIST results. The error bars are for the Round-Robin data and correspond to $\pm 2 s_{r}$. 


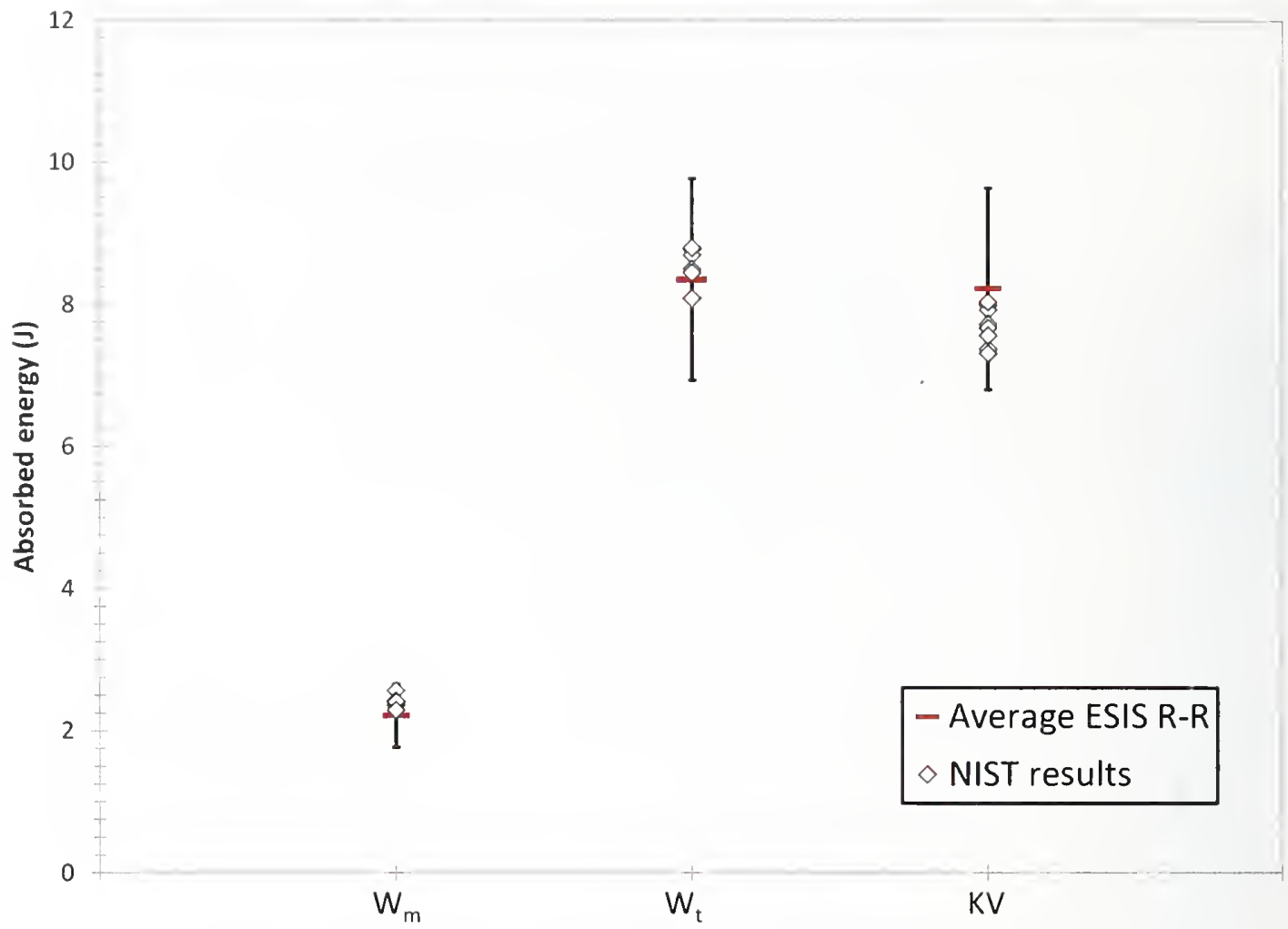

Figure 12 - Instrumented energy values: comparison between ESIS Round-Robin and NIST results. The error bars are for the Round-Robin data and correspond to $\pm 2 s_{r}$.

The results obtained at NIST are in excellent agreement with data from the ESIS TC5 Round-Robin, as shown by the comparisons with Round-Robin average values and $98 \%$ confidence intervals $\left( \pm 2 s_{r}\right.$ ). Based on these results, the NIST small-scale instrumented impact tester appears well-suited to perform the tests planned for this project. 


\section{Development of MCVN SRM's: preliminary testing of low, high and super-high KLST and RHS specimens}

\subsection{Materials and test matrix}

A number of broken (previously tested) full-size NIST verification specimens of different certified energy levels were selected for the fabrication of MCVN specimens.

For the low-energy level, tested specimens from lot LL-103 were selected. This lot has a certified absorbed energy value of $15.3 \mathrm{~J}$ (expanded uncertainty $0.1 \mathrm{~J}$ ) at $-40^{\circ} \mathrm{C}$. At the high-energy level, specimens from lot $\mathrm{HH}-103$ were used (certified absorbed energy: $97.5 \pm 0.6 \mathrm{~J}$ at $-40^{\circ} \mathrm{C}$ ). Both LL-103 and $\mathrm{HH}-103$ are also used as dynamic impact force verification specimens at room temperature (Standard Reference Materials 2113 and 2112. respectively). Their certified values for absorbed energy and maximum force at $21{ }^{\circ} \mathrm{C}$, established through an international round-robin exercise coordinated by NIST [16], are: $18.2 \mathrm{~J}$ and $33.00 \mathrm{kN}$ for LL-103 and $105.3 \mathrm{~J}$ and $24.06 \mathrm{kN}$ for HH-103. Both SRM 2112 and SRM 2113 are made from 4340 alloy steel.

For the super-high energy level, lot SH-36 was used. Lot SH-36 is made from T200 maraging steel. Its certified energy value at $21^{\circ} \mathrm{C}$ is $239.8 \mathrm{~J}$. No certified maximum force value was available, but the average value of $51 \mathrm{CVN}$ instrumented tests performed by NIST at $21^{\circ} \mathrm{C}$ on this material for a different study [17] was: $F_{m}=$ $25.64 \mathrm{kN}$, with a standard deviation $\sigma=0.09 \mathrm{kN}$. For the same 51 tests, performed on two different machines (TO2 and TO3) equipped with the same instrumented striker, the average value of $K V$ was $(239.04 \pm 5.97) \mathrm{kN}$.

For two energy levels (low and high), $32 \mathrm{MCVN}$ specimens for each KLST type (KLST and RHS) were extracted from broken full-size CVN samples. For super-high energy specimens, only eight specimens of each type were machined. The aim of testing super-high MCVN specimens in this preliminary phase was simply to establish whether absorbed energy values were significantly different from those obtained from high energy specimens. From every broken CVN half, four MCVN specimens could be obtained, as shown in Figure 13. As can be seen, the original orientations of the CVN specimen and notch were preserved.

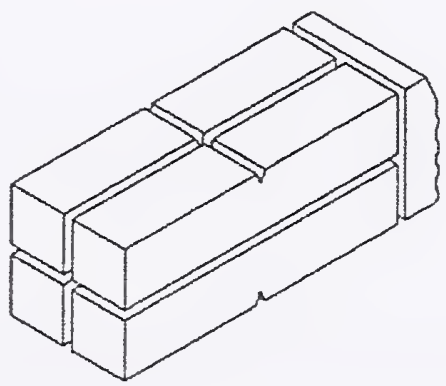

Figure 13 - Sketch of the extraction of four MCVN specimens from one broken CVN half.

The MCVN test matrix for the preliminary qualification phase is shown in Table 3.

Table 3 - Test matrix for the preliminary phase of KLST testing at NIST.

\begin{tabular}{|c|c|c|c|c|c|c|c|}
\hline \multirow{2}{*}{$\begin{array}{c}\text { Energy } \\
\text { level }\end{array}$} & \multirow{2}{*}{ Lot } & \multicolumn{3}{|c|}{ KLST specimens } & \multicolumn{3}{c|}{ RHS specimens } \\
\cline { 3 - 8 } & & Non-instrum. & Instrumented & Total & Non-instrum. & Instrumented & Total \\
\hline Low & LL-103 & 7 & 25 & 32 & 7 & 25 & 32 \\
\hline High & HH-103 & 7 & 25 & 32 & 7 & 25 & 32 \\
\hline Super-High & SH-36 & 3 & 5 & 8 & 3 & 5 & 8 \\
\hline
\end{tabular}


As indicated in Table 3, a few tests were performed non-instrumented.

\subsection{Low-energy MCVN test results}

Thirty-two KLST specimens and thirty-two RHS specimens from lot LL-103 were tested at room temperature. Force, displacement and absorbed energy results are collected in Table 4 (KLST) and Table 5 (RHS). For each parameter, the tables also provide the average value $\bar{A}$, the absolute standard deviation $\sigma$ and the percent coefficient of variation $\mathrm{CV}=\sigma / \bar{A} \times 100 \%$.

In Table 4 and Table 5, the subscripts $i u$ and $a$ indicate brittle fracture and crack arrest, respectively.

Table 4 - NIST test results from KLST specimens of LL-103 (low energy). Specimens 26-32 were tested non-instrumented.

\begin{tabular}{|c|c|c|c|c|c|c|c|c|c|c|c|c|c|c|c|c|c|}
\hline $\begin{array}{c}\text { Specimen } \\
\text { id }\end{array}$ & $\begin{array}{c}F_{E V} \\
(k N)\end{array}$ & $\begin{array}{c}F_{m} \\
(k N)\end{array}$ & $\begin{array}{c}F_{\text {iu }} \\
\langle\mathrm{kN}\rangle\end{array}$ & $\begin{array}{c}F_{\mathrm{a}} \\
(\mathrm{kN})\end{array}$ & $\begin{array}{c}s_{B V} \\
(\mathrm{~mm})\end{array}$ & $\begin{array}{c}s_{\mathrm{m}} \\
(\mathrm{mm})\end{array}$ & $\begin{array}{c}s_{\mathrm{tu}} \\
(\mathrm{mm})\end{array}$ & $\begin{array}{c}s_{\mathrm{a}} \\
(\mathrm{mm})\end{array}$ & $\begin{array}{c}s_{t} \\
(\mathrm{~mm})\end{array}$ & $\begin{array}{c}W_{B Y} \\
(J)\end{array}$ & $\begin{array}{l}W_{m} \\
(J)\end{array}$ & $\begin{array}{l}W_{\text {lu }} \\
\text { (J) }\end{array}$ & $\begin{array}{l}W_{\mathrm{a}} \\
(\mathrm{J})\end{array}$ & $\begin{array}{l}W_{t} \\
\text { (J) }\end{array}$ & $\begin{array}{l}K V \\
(J)\end{array}$ & $K V / W_{\mathrm{t}}$ & $\begin{array}{c}K V-W_{\mathrm{t}} \\
\text { (J) }\end{array}$ \\
\hline 1 & 2.21 & 2.65 & 2.62 & 1.31 & 0.37 & 0.59 & 0.59 & 0.61 & 0.96 & 0.44 & 0.96 & 0.96 & 1.00 & 1.27 & 1.34 & 1.055 & 0.07 \\
\hline 2 & 2.19 & 2.59 & 2.59 & 1.63 & 0.38 & 0.60 & 0.60 & 0.62 & 0.97 & 0.43 & 0.97 & 0.97 & 1.02 & 1.30 & 1.41 & 1.085 & 0.11 \\
\hline 3 & 2.16 & 2.62 & 2.62 & 1.27 & 0.37 & 0.63 & 0.63 & 0.69 & 1.01 & 0.41 & 1.04 & 1.04 & 1.14 & 1.33 & 1.38 & 1.038 & 0.05 \\
\hline 4 & 2.13 & 2.51 & 2.51 & 1.44 & 0.35 & 0.59 & 0.59 & 0.61 & 1.03 & 0.39 & 0.95 & 0.95 & 0.99 & 1.27 & 1.35 & 1.063 & 0.08 \\
\hline 5 & & & & & & & & & & & & & & & 1.48 & & \\
\hline 6 & 2.21 & 2.64 & 2.64 & 1.58 & 0.39 & 0.62 & 0.62 & 0.65 & 1.01 & 0.46 & 1.01 & 1.01 & 1.09 & 1.36 & 1.43 & 1.051 & 0.07 \\
\hline 7 & 2.20 & 2.61 & 2.57 & 1.53 & 0.36 & 0.60 & 0.63 & 0.65 & 1.05 & 0.41 & 1.02 & 1.10 & 1.14 & 1.40 & 1.46 & 1.043 & 0.06 \\
\hline 8 & 2.17 & 2.65 & 2.65 & 1.54 & 0.37 & 0.60 & 0.60 & 0.62 & 1.02 & 0.41 & 0.98 & 0.98 & 1.03 & 1.30 & 1.36 & 1.046 & 0.06 \\
\hline 9 & 2.19 & 2.63 & 2.59 & 1.45 & 0.36 & 0.62 & 0.63 & 0.66 & 0.93 & 0.42 & 1.05 & 1.08 & 1.15 & 1.37 & 1.44 & 1.051 & 0.07 \\
\hline 10 & 2.18 & 2.56 & 2.56 & 1.34 & 0.37 & 0.58 & 0.58 & 0.60 & 0.96 & 0.42 & 0.91 & 0.91 & 0.95 & 1.22 & 1.29 & 1.057 & 0.07 \\
\hline 11 & 2.15 & 2.58 & 2.53 & 1.59 & 0.37 & 0.62 & 0.65 & 0.67 & 1.02 & 0.40 & 1.02 & 1.10 & 1.15 & 1.39 & 1.45 & 1.043 & 0.06 \\
\hline 12 & 2.16 & 2.57 & 2.57 & 1.14 & 0.35 & 0.56 & 0.56 & 0.59 & 0.94 & 0.41 & 0.92 & 0.92 & 0.96 & 1.22 & 1.30 & 1.066 & 0.08 \\
\hline 13 & 2.16 & 2.65 & 2.60 & 1.28 & 0.37 & 0.62 & 0.63 & 0.66 & 1.01 & 0.42 & 1.03 & 1.05 & 1.12 & 1.33 & 1.39 & 1.045 & 0.06 \\
\hline 14 & 2.12 & 2.64 & 2.64 & 1.17 & 0.38 & 0.63 & 0.63 & 0.67 & 1.09 & 0.38 & 1.00 & 1.00 & 1.10 & 1.32 & 1.39 & 1.053 & 0.07 \\
\hline 15 & 2.24 & 2.50 & 2.50 & 1.48 & 0.37 & 0.56 & 0.56 & 0.61 & 0.93 & 0.46 & 0.93 & 0.93 & 0.99 & 1.21 & 1.25 & 1.033 & 0.04 \\
\hline 16 & 2.12 & 2.57 & 2.57 & 1.56 & 0.37 & 0.59 & 0.59 & 0.64 & 1.03 & 0.42 & 0.92 & 0.92 & 1.01 & 1.25 & 1.34 & 1.072 & 0.09 \\
\hline 17 & 2.19 & 2.64 & 2.64 & 1.12 & 0.37 & 0.61 & 0.61 & 0.63 & 0.97 & 0.42 & 1.00 & 1.00 & 1.04 & 1.28 & 1.37 & 1.070 & 0.09 \\
\hline 18 & 2.03 & 2.51 & 2.51 & 1.44 & 0.35 & 0.57 & 0.57 & 0.62 & 0.94 & 0.37 & 0.86 & 0.86 & 0.95 & 1.18 & 1.28 & 1.085 & 0.10 \\
\hline 19 & 2.18 & 2.66 & 2.66 & 1.22 & 0.37 & 0.59 & 0.59 & 0.61 & 0.95 & 0.43 & 0.95 & 0.95 & 0.99 & 1.25 & 1.33 & 1.064 & 0.08 \\
\hline 20 & 2.20 & 2.61 & 2.61 & 1.56 & 0.36 & 0.57 & 0.57 & 0.59 & 0.87 & 0.42 & 0.91 & 0.91 & 0.96 & 1.19 & & & \\
\hline 21 & 2.18 & 2.58 & 2.58 & 1.29 & 0.38 & 0.63 & 0.63 & 0.66 & 1.00 & 0.43 & 1.03 & 1.03 & 1.09 & 1.33 & 1.39 & 1.045 & 0.06 \\
\hline 22 & 2.16 & 2.61 & 2.51 & 1.23 & 0.37 & 0.62 & 0.63 & 0.65 & 0.92 & 0.41 & 1.00 & 1.03 & 1.07 & 1.28 & 1.37 & 1.070 & $0 . \infty 9$ \\
\hline 23 & 2.18 & 2.58 & 2.58 & 1.31 & 0.37 & 0.61 & 0.61 & 0.63 & 0.98 & 0.41 & 1.01 & 1.01 & 1.05 & 1.27 & 1.34 & 1.055 & 0.07 \\
\hline 24 & 2.15 & 2.57 & 2.57 & 1.41 & 0.37 & 0.61 & 0.61 & 0.63 & 1.14 & 0.40 & 0.97 & 0.97 & 1.01 & 1.32 & 1.41 & 1.068 & 0.09 \\
\hline 25 & 2.16 & 2.61 & 2.52 & 1.24 & 0.37 & 0.61 & 0.62 & 0.65 & 1.00 & 0.43 & 1.00 & 1.03 & 1.09 & 1.34 & 1.43 & 1.067 & 0.09 \\
\hline 26 & & & & & & & & & & & & & & & 1.33 & & \\
\hline 27 & & & & & & & & & & & & & & & 1.33 & & \\
\hline 28 & & & & & & & & & & & & & & & 1.29 & & \\
\hline 29 & & & & & & & & & & & & & & & 1.42 & & \\
\hline 30 & & & & & & & & & & & & & & & 1.39 & & \\
\hline 31 & & & & & & & & & & & & & & & 1.27 & & \\
\hline 32 & & & & & & & & & & & & & & & & & \\
\hline$N$ & 24 & 24 & 24 & 24 & 24 & 24 & 24 & 24 & 24 & 24 & 24 & 24 & 24 & 24 & 30 & 23 & 23 \\
\hline Average & 2.17 & 2.60 & 2.58 & 1.38 & 0.37 & 0.60 & 0.61 & 0.63 & 0.99 & 0.42 & 0.98 & 0.99 & 1.05 & 1.29 & 1.37 & 1.058 & 0.07 \\
\hline$\sigma(\mathrm{abs})$ & 0.041 & 0.046 & 0.049 & 0.156 & 0.010 & 0.022 & 0.026 & 0.027 & 0.058 & 0.021 & 0.049 & 0.063 & 0.067 & 0.061 & 0.060 & 0.014 & 0.017 \\
\hline $\mathrm{CV}$ & $1.9 \%$ & $1.8 \%$ & $1.9 \%$ & $11.3 \%$ & $2.6 \%$ & $3.6 \%$ & $4.2 \%$ & $4.3 \%$ & $5.9 \%$ & $5.0 \%$ & $5.1 \%$ & $6.4 \%$ & $6.4 \%$ & $4.7 \%$ & $4.4 \%$ & $1.3 \%$ & $22.5 \%$ \\
\hline
\end{tabular}

NOTE - $N$ is the number of available data. Instrumented data for specimen 5 were lost because the acquisition system did not trigger. The values of $K V$ for specimens 20 and 32 were lost because the optical encoder did not trigger. 
Table 5 - NIST test results from RHS specimens of LL-103 (low energy). Specimens 26-32 were tested non-instrumented.

\begin{tabular}{|c|c|c|c|c|c|c|c|c|c|c|c|c|c|c|c|c|c|}
\hline $\begin{array}{c}5 \text { pecimen } \\
\text { id }\end{array}$ & $\begin{array}{c}F_{g y} \\
(k N)\end{array}$ & $\begin{array}{c}F_{m} \\
(k N)\end{array}$ & $\begin{array}{c}F_{10} \\
(k N)\end{array}$ & $\begin{array}{c}F_{\mathrm{a}} \\
(\mathrm{kN})\end{array}$ & $\begin{array}{c}\mathrm{s}_{g y} \\
(\mathrm{~mm})\end{array}$ & $\begin{array}{c}\mathrm{s}_{\mathrm{m}} \\
(\mathrm{mm})\end{array}$ & $\begin{array}{c}s_{w} \\
(\mathrm{~mm})\end{array}$ & $\begin{array}{c}\mathrm{s}_{\mathrm{a}} \\
(\mathrm{mm})\end{array}$ & $\begin{array}{c}\mathrm{s}_{\mathrm{t}} \\
(\mathrm{mm})\end{array}$ & $\begin{array}{l}W_{g Y} \\
(J)\end{array}$ & $\begin{array}{l}W_{m} \\
\text { (J) }\end{array}$ & $\begin{array}{l}W_{10} \\
\text { (J) }\end{array}$ & $\begin{array}{l}W_{a} \\
\text { (J) }\end{array}$ & $\begin{array}{l}W_{t} \\
(J)\end{array}$ & $\begin{array}{l}\text { KV } \\
\text { (J) }\end{array}$ & $K V / W_{1}$ & $\begin{array}{l}K V-W_{i} \\
\text { (J) }\end{array}$ \\
\hline 1 & 6.06 & 8.20 & 8.20 & 2.35 & 0.25 & 0.52 & 0.52 & 0.55 & 0.71 & 0.81 & 2.75 & 2.75 & 2.93 & 3.18 & 3.27 & 1.028 & 0.09 \\
\hline 2 & 5.98 & 8.24 & 8.12 & 1.55 & 0.25 & 0.54 & 0.55 & 0.59 & 0.96 & 0.78 & 2.90 & 2.98 & 3.20 & 3.50 & 3.55 & 1.014 & 0.05 \\
\hline 3 & 6.51 & 8.21 & 8.21 & 3.21 & 0.28 & 0.55 & 0.55 & 0.58 & 1.83 & 0.96 & 2.96 & 2.96 & 3.14 & 3.79 & 3.56 & 0.939 & -0.23 \\
\hline 4 & 6.02 & 8.26 & 8.26 & 2.83 & 0.26 & 0.53 & 0.53 & 0.56 & 0.83 & 0.77 & 2.79 & 2.79 & 2.98 & 3.30 & 3.31 & 1.003 & 0.01 \\
\hline 5 & 5.93 & 8.21 & 7.73 & 3.35 & 0.26 & 33.88 & 0.50 & 0.53 & 0.88 & 0.75 & 2.35 & 2.51 & 2.69 & 3.07 & 3.09 & 1.007 & 0.02 \\
\hline 6 & 6.09 & 8.13 & 8.13 & 3.21 & 0.25 & 0.53 & 0.53 & 0.56 & 1.83 & 0.81 & 2.83 & 2.83 & 3.02 & 3.70 & 3.45 & 0.932 & -0.25 \\
\hline 7 & 5.91 & 8.16 & 8.16 & 2.97 & 0.26 & 0.54 & 0.54 & 0.57 & 0.72 & 0.73 & 2.81 & 2.81 & 3.00 & 3.26 & 3.37 & 1.034 & 0.11 \\
\hline 8 & 6.06 & 8.10 & 8.10 & 1.87 & 0.25 & 0.53 & 0.53 & 0.56 & 0.91 & 0.79 & 2.83 & 2.83 & 2.99 & 3.31 & 3.29 & 0.994 & -0.02 \\
\hline 9 & 6.06 & 8.30 & 8.30 & 2.00 & 0.25 & 0.53 & 0.53 & 0.57 & 0.92 & 0.77 & 2.88 & 2.88 & 3.11 & 3.44 & 3.45 & 1.003 & 0.01 \\
\hline 10 & 6.03 & 8.42 & 8.42 & 1.93 & 0.24 & 0.52 & 0.52 & 0.56 & 0.71 & 0.74 & 2.86 & 2.86 & 3.11 & 3.33 & 3.39 & 1.018 & 0.06 \\
\hline 11 & 6.41 & 8.22 & 8.22 & 2.29 & 0.30 & 0.59 & 0.59 & 0.62 & 1.88 & 0.92 & 3.09 & 3.09 & 3.25 & 3.91 & 3.65 & 0.934 & -0.26 \\
\hline 12 & 5.97 & 8.34 & 8.16 & 2.12 & 0.25 & 0.54 & 0.55 & 0.60 & 1.87 & 0.77 & 2.87 & 2.96 & 3.26 & 4.01 & 3.74 & 0.933 & -0.27 \\
\hline 13 & 5.07 & 8.29 & 8.29 & 3.03 & 0.23 & 0.51 & 0.51 & 0.54 & 181 & 0.78 & 2.82 & 2.82 & 3.00 & 3.63 & 3.44 & 0.948 & -0.19 \\
\hline 14 & 6.08 & 8.03 & 8.03 & 2.11 & 0.25 & 0.53 & 0.53 & 0.56 & 1.82 & 0.80 & 2.83 & 2.83 & 2.99 & 3.65 & 3.44 & 0.942 & -0.21 \\
\hline 15 & 5.96 & 7.86 & 7.83 & 2.91 & 0.25 & 0.49 & 0.50 & 0.53 & 0.88 & 0.75 & 2.50 & 2.58 & 2.75 & 3.11 & 3.11 & 1.000 & 0.00 \\
\hline 16 & 5.93 & 7.98 & 7.89 & 2.89 & 0.25 & 0.49 & 0.51 & 0.54 & 1.81 & 0.77 & 2.46 & 2.62 & 2.80 & 3.42 & 3.44 & 1.006 & 0.02 \\
\hline 17 & 6.01 & 8.27 & 8.01 & 2.16 & 0.29 & 0.56 & 0.57 & 0.60 & 1.85 & 0.84 & 2.86 & 2.94 & 3.10 & 3.65 & 3.42 & 0.937 & -0.23 \\
\hline 18 & 6.13 & 7.98 & 7.98 & 176 & 0.24 & 0.51 & 0.51 & 0.54 & 0.89 & 0.81 & 2.77 & 2.77 & 2.92 & 3.23 & 3.20 & 0.991 & -0.03 \\
\hline 19 & 6.15 & 8.27 & 8.05 & 1.53 & 0.25 & 0.55 & 0.56 & 0.59 & 0.75 & 0.78 & 3.08 & 3.16 & 3.31 & 3.54 & 3.55 & 1.003 & 0.01 \\
\hline 20 & 5.98 & 8.04 & 8.04 & 1.69 & 0.25 & 0.50 & 0.50 & 0.54 & 0.88 & 0.75 & 2.60 & 2.60 & 2.83 & 3.16 & 3.13 & 0.991 & -0.03 \\
\hline 21 & 6.09 & 8.13 & 7.91 & 2.30 & 0.25 & 0.51 & 0.52 & 0.55 & 182 & 0.76 & 2.69 & 2.78 & 2.93 & 3.61 & 3.39 & 0.939 & -0.22 \\
\hline 22 & 6.05 & 8.27 & 8.27 & 2.06 & 0.26 & 0.54 & 0.54 & 0.58 & 1.52 & 0.80 & 2.84 & 2.84 & 3.08 & 3.60 & 3.44 & 0.956 & -0.16 \\
\hline 23 & 6.04 & 8.41 & 8.41 & 168 & 0.25 & 0.53 & 0.53 & 0.57 & 0.71 & 0.80 & 2.84 & 2.84 & 3.08 & 3.30 & 3.34 & 1.012 & 0.04 \\
\hline 24 & 6.01 & 8.12 & 8.12 & 2.66 & 0.25 & 0.52 & 0.52 & 0.55 & 0.70 & 0.79 & 2.73 & 2.73 & 2.91 & 3.16 & 3.26 & 1.032 & 0.10 \\
\hline 25 & 6.06 & 8.07 & 8.07 & 2.22 & 0.26 & 0.53 & 0.53 & 0.56 & 0.91 & 0.78 & 2.80 & 2.80 & 2.96 & 3.31 & 3.29 & 0.994 & -0.02 \\
\hline 26 & & & & & & & & & & & & & & & 3.43 & & \\
\hline 27 & & & & & & & & & & & & & & & 3.40 & & \\
\hline 28 & & & & & & & & & & & & & & & 3.61 & & \\
\hline 29 & & & & & & & & & & & & & & & 3.71 & & \\
\hline 30 & & & & & & & & & & & & & & & 3.65 & & \\
\hline 31 & & & & & & & & & & & & & & & 3.52 & & \\
\hline 32 & & & & & & & & & & & & & & & 3.67 & & \\
\hline$N$ & 25 & 25 & 25 & 25 & 25 & 25 & 25 & 25 & 25 & 25 & 25 & 25 & 25 & 25 & 32 & 25 & 25 \\
\hline Average & 6.06 & 8.18 & 8.12 & 2.35 & 0.26 & 1.86 & 0.53 & 0.56 & 1.22 & 0.79 & 2.79 & 2.82 & 3.01 & 3.45 & 3.38 & 0.984 & -0.06 \\
\hline$\sigma(a b s)$ & 0.135 & 0.137 & 0.170 & 0.562 & 0.015 & 6.670 & 0.022 & 0.023 & 0.499 & 0.051 & 0.169 & 0.149 & 0.156 & 0.255 & 0.161 & 0.035 & 0.129 \\
\hline$C V$ & $2.2 \%$ & $1.7 \%$ & $2.1 \%$ & $24.0 \%$ & $5.9 \%$ & $358.1 \%$ & $4.2 \%$ & $4.1 \%$ & $41.1 \%$ & $6.5 \%$ & $6.1 \%$ & $5.3 \%$ & $5.2 \%$ & $7.4 \%$ & $4.8 \%$ & $3.6 \%$ & $-202.3 \%$ \\
\hline
\end{tabular}

NOTE $-N$ is the number of available data.

The variation of MCVN results was compared to the scatter resulting from room temperature pilot lot tests with the three NIST reference machines (TO2, TK and TO3). The coefficients of variation for $K V$ measured from the three machines on the basis of 30, 25 and 25 tests, respectively, are: $3.75 \%$ (TO2), 3.46\% (TK) and $2.22 \%$ (TO3). The corresponding CV values from KLST and RHS tests, $4.4 \%$ and $4.8 \%$, are slightly higher. Note that the coefficients of variation for absorbed energy calculated from the instrumented force/displacement curves $\left(W_{t}\right)$ are higher for both KLST tests $(4.7 \%)$ and RHS tests $(7.4 \%)$. On the other hand, force-based parameters from MCVN tests have lower $\mathrm{CV}$ values (about $2 \%$ ), with the exception of arrest forces $F_{a}$.

\subsubsection{Calculation of sample size and outlier analysis}

The sample size, $n$, is the minimum number of specimens from a given production lot that should be tested in a verification test [18]. It is calculated as:

$$
n=\left(\frac{3 s_{p}}{E}\right)^{2}
$$


where $s_{p}$ is the pooled standard deviation (or the machine standard deviation if only one maehine is used), and $E$ is the greatest of $0.105 \mathrm{~J}$ (KLST) or $0.263 \mathrm{~J}$ (RHS) ${ }^{\dagger}$ and $5 \%$ of the mean absorbed energy. For KLST and RHS speeimens of LL-103, the sample size is 2.9 and 3.8, respectively. For eomparison, the testing of the pilot lot of LL-103 full-size speeimens returned a sample size of 1.3 at $-40{ }^{\circ} \mathrm{C}$ and 1.6 at room temperature.

The outlier analysis is eonventionally performed by means of box-and-whiskers plots to provide a graphical summary of the data and identify outliers, defined as values that are lower than the first quartile or higher than the third quartile by more than 1.5 times the absolute differenee between the first and third quartiles. If a lot has more than $5 \%$ outliers, it may be rejeeted [18]. It is important to note than a speeimen identified as an outlier is not removed from the analysis, unless it shows physical evidence of jamming, material flaws, or other atypieal behavior. Box-and-whiskers plots for $K V$ values are shown in Figure 14 (KLST) and Figure 15 (RHS). No outliers were deteeted.



Figure 14 - Box-and-whisker plot for $K V$ values from KLST specimens of LL-103.

In addition to the box-and-whiskers plot method, the outlier analysis was also eonducted aecording to Grubbs' test, also known as the maximum normed residual test or the extreme studentized deviate method [19]. This statistical test is used for data sets that are assumed to eome from a normally distributed population, and should not be used for sample sizes of six or less. Grubbs' test deteets one outlier at a time, and should be repeated onee the first outlier (if deteeted) is expunged from the data set.

\footnotetext{
${ }^{+} T$ The NIST procedure for standard Charpy specimens [18] uses the greater between $1.4 \mathrm{~J}$ and $5 \%$ of $K V$ for the factor $E$ in Eq. (1). In this study, the value $1.4 \mathrm{~J}$ was normalized by the ratio between $K V_{\text {full-size }}$ and $K V_{\text {sub-size }}$ for the specific energy level and MCVN type, i.e., 13.34 for KLST and 5.33 for RHS at the low energy level (see section 5.2).
} 
Grubbs' test was applied to values of $F_{\mathrm{gy}}, F_{\mathrm{m}}, W_{\mathrm{m}}, W_{\mathrm{t}}$ and $K V$ and calculated from the tests performed. Outlier detection was carried out at a significance level $\alpha=0.01$. The results for MCVN specimens of LL-103 are summarized in Table 6.

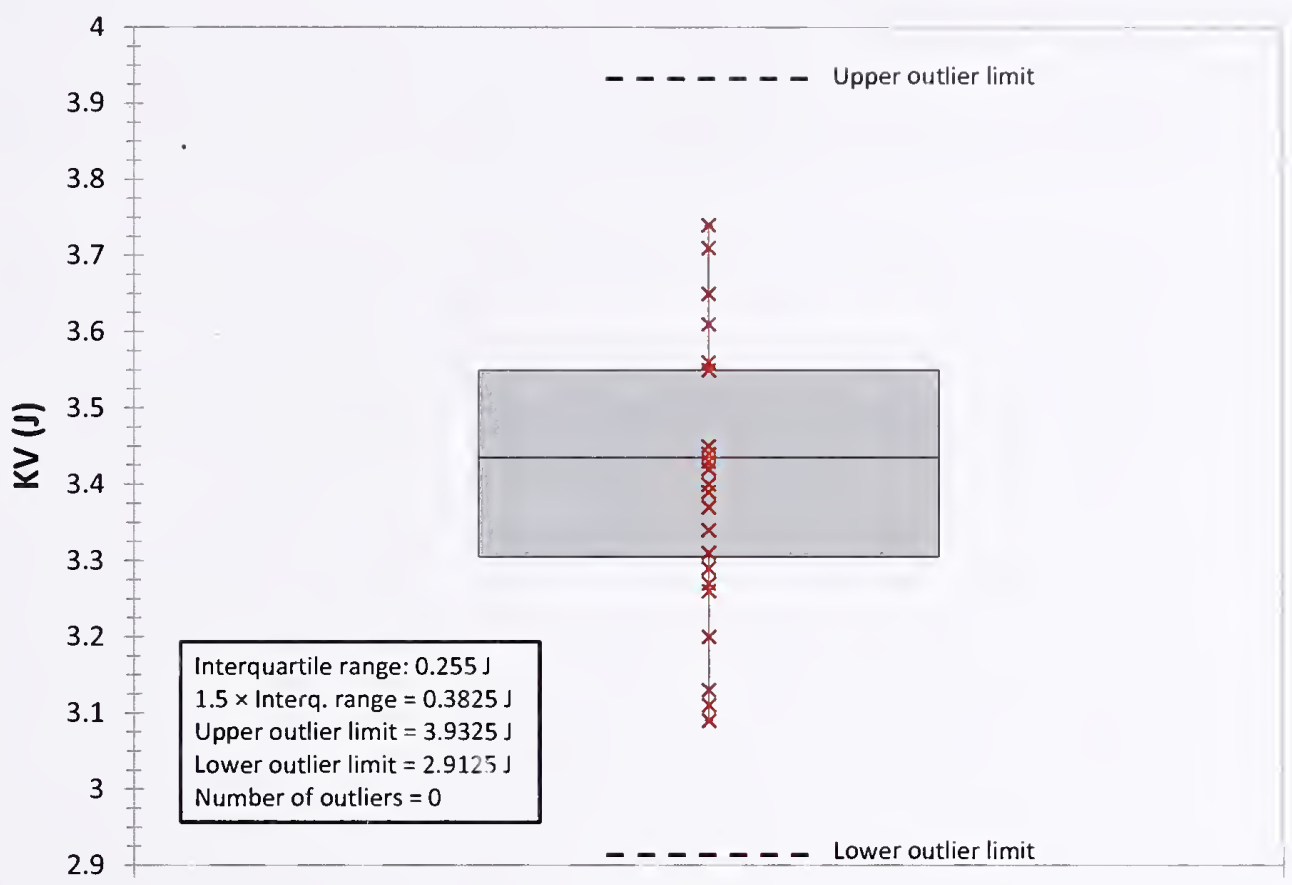

LL-103

Figure 15 - Box-and-whisker plot for $K V$ values from RHS specimens of LL-103.

Table 6 - Results of Grubbs' test for the detection of outliers on MCVN tests of LL-103. $N$ is the number of values analyzed.

\begin{tabular}{|c|c|c|c|c|}
\hline $\begin{array}{c}\text { Specimen } \\
\text { type }\end{array}$ & Parameter & $N$ & $\begin{array}{c}\text { Outliers } \\
\text { detected }\end{array}$ & $\begin{array}{c}\text { Outlier } \\
\text { specimens }^{\ddagger}\end{array}$ \\
\hline & $F_{\mathrm{gy}}$ & 24 & 1 & 18 \\
& $F_{\mathrm{m}}$ & 24 & 0 & - \\
KLST & $W_{\mathrm{m}}$ & 24 & 0 & - \\
& $W_{\mathrm{t}}$ & 24 & 0 & - \\
& $K V$ & 30 & 0 & - \\
\hline \multirow{5}{*}{ RHS } & $F_{\mathrm{gy}}$ & 25 & 2 & $3,11^{\S}$ \\
& $F_{\mathrm{m}}$ & 25 & 0 & - \\
& $W_{\mathrm{m}}$ & 25 & 0 & - \\
& $W_{\mathrm{t}}$ & 25 & 0 & - \\
& $K V$ & 32 & 0 & - \\
\hline
\end{tabular}

Outliers were detected only for forces at general yield. This is not surprising, considering the intrinsic subjectivity of the determination of the general yield point from the instrumented test record. For $K V$ values, the

\$ See Table 4 (KLST) and Table 5 (RHS) for specimen numbers.

${ }^{\S}$ The two outliers were detected in two successive runs of Grubbs' test. 
outeome of the Grubbs' test eonfirms the results of the box-and-whisker method (i.e., no outliers deteeted at $\alpha=$ $0.01)$.

\subsection{High-energy MCVN test results}

Thirty-two KLST speeimens and thirty-two RHS specimens from lot HH-103 were tested at room temperature. Foree, displacement and absorbed energy results are collected in Table 7 (KLST) and Table 8 (RHS).

Table 7 - NIST test results from KLST specimens of HH-103 (high energy). Specimens 26-32 were tested non-instrumented.

\begin{tabular}{|c|c|c|c|c|c|c|c|c|c|c|c|}
\hline $\begin{array}{c}\text { 5pecimen } \\
\text { id }\end{array}$ & $\begin{array}{c}F_{B Y} \\
(k N)\end{array}$ & $\begin{array}{c}F_{m} \\
(k N)\end{array}$ & $\begin{array}{c}s_{B Y} \\
(\mathrm{~mm})\end{array}$ & $\begin{array}{c}s_{\mathrm{m}} \\
(\mathrm{mm})\end{array}$ & $\begin{array}{c}s_{t} \\
(\mathrm{~mm})\end{array}$ & $\begin{array}{l}W_{g V} \\
(J)\end{array}$ & $\begin{array}{l}W_{\mathrm{m}} \\
(J)\end{array}$ & $\begin{array}{l}W_{t} \\
(J)\end{array}$ & $\begin{array}{l}K V \\
\text { (J) }\end{array}$ & $K V / W_{t}$ & $\begin{array}{c}W_{t}-K V \\
\text { (J) }\end{array}$ \\
\hline 1 & 1.49 & 1.91 & 0.28 & 0.65 & 12.35 & 0.21 & 0.86 & 5.77 & 5.09 & 0.882 & 0.68 \\
\hline 2 & 1.50 & 1.92 & 0.26 & 0.63 & 13.10 & 0.21 & 0.87 & 5.95 & 5.25 & 0.882 & 0.70 \\
\hline 3 & 1.51 & 1.94 & 0.28 & 0.64 & 11.68 & 0.22 & 0.87 & 5.96 & 5.29 & 0.888 & 0.67 \\
\hline 4 & 1.51 & 1.92 & 0.27 & 0.62 & 12.22 & 0.21 & 0.85 & 5.85 & 5.15 & 0.880 & 0.70 \\
\hline 5 & 1.50 & 1.90 & 0.29 & 0.66 & 12.13 & 0.21 & 0.89 & 5.70 & 5.00 & 0.877 & 0.70 \\
\hline 6 & 1.49 & 1.91 & 0.27 & 0.64 & 12.20 & 0.23 & 0.86 & 5.91 & 5.13 & 0.868 & 0.78 \\
\hline 7 & 1.53 & 1.92 & 0.27 & 0.62 & 11.49 & 0.24 & 0.86 & 6.02 & 5.31 & 0.882 & 0.71 \\
\hline 8 & 1.50 & 1.90 & 0.26 & 0.63 & 11.59 & 0.20 & 0.88 & 6.03 & 5.28 & 0.876 & 0.75 \\
\hline 9 & 1.50 & 1.90 & 0.28 & 0.63 & 12.18 & 0.21 & 0.83 & 5.81 & 5.11 & 0.880 & 0.70 \\
\hline 10 & 1.49 & 1.91 & 0.27 & 0.64 & 12.17 & 0.21 & 0.86 & 5.86 & 5.13 & 0.875 & 0.73 \\
\hline 11 & 1.51 & 1.92 & 0.26 & 0.62 & 12.18 & 0.21 & 0.85 & 6.14 & 5.38 & 0.876 & 0.76 \\
\hline 12 & 1.49 & 1.93 & 0.26 & 0.62 & 11.41 & 0.20 & 0.86 & 5.92 & 5.26 & 0.889 & 0.66 \\
\hline 13 & 1.48 & 1.92 & 0.28 & 0.65 & 12.19 & 0.20 & 0.87 & 5.84 & 5.12 & 0.877 & 0.72 \\
\hline 14 & 1.48 & 1.90 & 0.27 & 0.63 & 11.66 & 0.21 & 0.86 & 5.78 & 5.10 & 0.882 & 0.68 \\
\hline 15 & 1.49 & 1.91 & 0.28 & 0.64 & 11.47 & 0.21 & 0.86 & 5.99 & 5.34 & 0.891 & 0.65 \\
\hline 16 & 1.54 & 1.93 & 0.28 & 0.65 & 12.37 & 0.22 & 0.89 & 5.79 & 5.10 & 0.881 & 0.69 \\
\hline 17 & 1.51 & 1.93 & 0.28 & 0.63 & 12.29 & 0.22 & 0.86 & 5.94 & 5.18 & 0.872 & 0.76 \\
\hline 18 & 1.52 & 1.91 & 0.28 & 0.63 & 12.11 & 0.22 & 0.86 & 6.11 & 5.33 & 0.872 & 0.78 \\
\hline 19 & 1.49 & 1.91 & 0.28 & 0.64 & 12.20 & 0.21 & 0.86 & 5.88 & 5.14 & 0.874 & 0.74 \\
\hline 20 & 1.52 & 1.93 & 0.28 & 0.65 & 11.59 & 0.21 & 0.88 & 6.03 & 5.28 & 0.876 & 0.75 \\
\hline 21 & 1.45 & 1.90 & 0.26 & 0.63 & 11.50 & 0.20 & 0.86 & 5.87 & 5.21 & 0.888 & 0.66 \\
\hline 22 & 1.49 & 1.91 & 0.26 & 0.62 & 12.18 & 0.20 & 0.85 & 5.74 & 5.10 & 0.889 & 0.64 \\
\hline 23 & 1.54 & 1.92 & 0.28 & 0.63 & 11.65 & 0.25 & 0.85 & 5.76 & 5.10 & 0.885 & 0.66 \\
\hline 24 & 1.52 & 1.91 & 0.29 & 0.65 & 12.20 & 0.23 & 0.88 & 5.93 & 5.18 & 0.874 & 0.75 \\
\hline 25 & 1.61 & 1.93 & 0.33 & 0.67 & 11.52 & 0.26 & 0.89 & 6.08 & 5.34 & 0.878 & 0.74 \\
\hline 26 & & & & & & & & & 5.13 & & \\
\hline 27 & & & & & & & & & 5.15 & & \\
\hline 28 & & & & & & & & & 5.35 & & \\
\hline 29 & & & & & & & & & 5.36 & & \\
\hline 30 & & & & & & & & & 5.17 & & \\
\hline 31 & & & & & & & & & 5.29 & & \\
\hline 32 & & & & & & & & & 5.06 & & \\
\hline$N$ & 25 & 25 & 25 & 25 & 25 & 25 & 25 & 25 & 32 & 25 & 25 \\
\hline Average & 1.51 & 1.92 & 0.28 & 0.64 & 11.99 & 0.22 & 0.86 & 5.91 & 5.20 & 0.880 & 0.71 \\
\hline$\sigma(a b s)$ & 0.029 & 0.012 & 0.015 & 0.013 & 0.406 & 0.016 & 0.014 & 0.120 & 0.104 & 0.006 & 0.042 \\
\hline CV & $2.0 \%$ & $0.6 \%$ & $5.3 \%$ & $2.1 \%$ & $3.4 \%$ & $7.2 \%$ & $1.7 \%$ & $2.0 \%$ & $2.0 \%$ & $0.7 \%$ & $5.9 \%$ \\
\hline
\end{tabular}

The coeffieients of variation for $K V$ values obtained from the pilot lot testing of $\mathrm{HH}-103$ on the three NIST reference maehines were: $2.47 \%$ (TO2), $2.64 \%$ (TK) and $2.30 \%$ (TO3). Twenty-five full-size Charpy specimens were tested on each machine. The corresponding CV values from KLST and RHS tests, $2.0 \%$ and $2.6 \%$, respectively, are fully comparable. Unlike low-energy MCVN tests, the eoeffieients of variation for instrumented absorbed energies $\left(W_{\mathrm{t}}\right)$ are the same for KLST tests $(2.0 \%)$ and lower for RHS tests $(2.2 \%)$. Forees at general yield $\left(F_{\mathrm{gy}}\right)$ have comparable values of CV (about $2 \%$ ), while maximum foree values $\left(F_{\mathrm{m}}\right)$ exhibit very low variability $(\mathrm{CV}=0.6 \%)$. 
Table 8 - NIST test results from RHS specimens of HH-103 (high energy). Specimens 26-32 were tested non-instrumented.

\begin{tabular}{|c|c|c|c|c|c|c|c|c|c|c|c|}
\hline $\begin{array}{c}\text { Specimen } \\
\text { id }\end{array}$ & $\begin{array}{c}F_{g \gamma} \\
(\mathrm{kN})\end{array}$ & $\begin{array}{c}F_{m} \\
(k N)\end{array}$ & $\begin{array}{c}s_{g y} \\
(\mathrm{~mm})\end{array}$ & $\begin{array}{c}s_{\mathrm{m}} \\
(\mathrm{mm})\end{array}$ & $\begin{array}{c}s_{t} \\
(\mathrm{~mm})\end{array}$ & $\begin{array}{c}W_{g y} \\
(J)\end{array}$ & $\begin{array}{l}W_{m} \\
\text { (J) }\end{array}$ & $\begin{array}{l}W_{t} \\
(J)\end{array}$ & $\begin{array}{l}K V \\
(J)\end{array}$ & $K V / W_{t}$ & $\begin{array}{c}W_{t}-K V \\
(J)\end{array}$ \\
\hline 1 & 5.00 & 6.34 & 0.20 & 0.92 & 29.29 & 0.52 & 4.84 & 16.14 & 14.45 & 0.895 & 1.69 \\
\hline 2 & 5.45 & 6.39 & 0.26 & 0.93 & 29.24 & 0.71 & 4.84 & 16.28 & 14.56 & 0.894 & 1.72 \\
\hline 3 & 5.28 & 6.41 & 0.25 & 0.91 & 29.30 & 0.65 & 4.71 & 16.12 & 14.40 & 0.893 & 1.72 \\
\hline 4 & 5.40 & 6.30 & 0.26 & 0.88 & 29.44 & 0.70 & 4.47 & 15.70 & 14.32 & 0.912 & 1.38 \\
\hline 5 & 5.36 & 6.39 & 0.25 & 0.92 & 29.31 & 0.70 & 4.75 & 16.09 & 14.41 & 0.896 & 1.68 \\
\hline 6 & 5.40 & 6.43 & 0.24 & 0.95 & 28.62 & 0.68 & 5.07 & 17.19 & 15.60 & 0.908 & 1.59 \\
\hline 7 & 5.23 & 6.39 & 0.24 & 0.93 & 10.30 & 0.64 & 4.80 & 16.45 & & & \\
\hline 8 & 5.34 & 6.36 & 0.25 & 0.91 & 29.30 & 0.68 & 4.66 & 16.08 & 14.51 & 0.902 & 1.57 \\
\hline 9 & 5.46 & 6.38 & 0.27 & 0.89 & 29.33 & 0.72 & 4.54 & 15.99 & 14.56 & 0.911 & 1.43 \\
\hline 10 & 5.37 & 6.35 & 0.24 & 0.88 & 29.52 & 0.67 & 4.58 & 15.49 & 13.78 & 0.890 & 1.71 \\
\hline 11 & 5.34 & 6.29 & 0.24 & 0.88 & 29.40 & 0.71 & 4.53 & 15.77 & 14.39 & 0.912 & 1.38 \\
\hline 12 & 5.37 & 6.32 & 0.24 & 0.90 & 29.13 & 0.67 & 4.70 & 16.51 & 14.99 & 0.908 & 1.52 \\
\hline 13 & 5.32 & 6.41 & 0.24 & 0.93 & 29.29 & 0.65 & 4.89 & 16.17 & 14.41 & 0.891 & 1.76 \\
\hline 14 & 5.43 & 6.33 & 0.28 & 0.94 & 29.41 & 0.71 & 4.70 & 15.73 & 14.15 & 0.900 & 1.58 \\
\hline 15 & 5.39 & 6.41 & 0.27 & 0.92 & 29.37 & 0.70 & 4.64 & 15.95 & 14.26 & 0.894 & 1.69 \\
\hline 16 & 5.52 & 6.39 & 0.31 & 0.96 & 27.39 & 0.84 & 4.84 & 16.61 & 15.05 & 0.906 & 1.56 \\
\hline 17 & 5.36 & 6.39 & 0.25 & 0.91 & 29.38 & 0.71 & 4.64 & 15.90 & 14.24 & 0.896 & 1.66 \\
\hline 18 & 5.37 & 6.37 & 0.26 & 0.90 & 29.44 & 0.71 & 4.58 & 15.75 & 14.11 & 0.896 & 1.64 \\
\hline 19 & 5.38 & 6.38 & 0.27 & 0.94 & 29.27 & 0.74 & 4.85 & 16.16 & 14.73 & 0.912 & 1.43 \\
\hline 20 & 5.38 & 6.44 & 0.26 & 0.96 & 29.20 & 0.72 & 4.98 & 16.36 & 14.67 & 0.897 & 1.69 \\
\hline 21 & 5.39 & 6.38 & 0.25 & 0.90 & 29.29 & 0.75 & 4.62 & 16.10 & 14.66 & 0.911 & 1.44 \\
\hline 22 & 5.40 & 6.36 & 0.26 & 0.91 & 10.56 & 0.69 & 4.68 & 16.13 & 14.74 & 0.914 & 1.39 \\
\hline 23 & 5.46 & 6.39 & 0.24 & 0.87 & 29.35 & 0.73 & 4.55 & 15.92 & 14.51 & 0.911 & 1.41 \\
\hline 24 & 5.55 & 6.43 & 0.21 & 0.83 & 29.22 & 0.74 & 4.60 & 16.29 & 14.69 & 0.902 & 1.60 \\
\hline 25 & 5.34 & 6.30 & 0.25 & 0.89 & 29.47 & 0.66 & 4.48 & 15.68 & 14.01 & 0.893 & 1.67 \\
\hline 26 & & & & & & & & & 14.48 & & \\
\hline 27 & & & & & & & & & 14.25 & & \\
\hline 28 & & & & & & & & & 14.56 & & \\
\hline 29 & & & & & & & & & 14.12 & & \\
\hline 30 & & & & & & & & & 14.07 & & \\
\hline 31 & & & & & & & & & 14.43 & & \\
\hline 32 & & & & & & & & & 15.23 & & \\
\hline$N$ & 25 & 25 & 25 & 25 & 25 & 25 & 25 & 25 & 31 & 24 & 24 \\
\hline Average & 5.37 & 6.37 & 0.25 & 0.91 & 27.71 & 0.70 & 4.70 & 16.10 & 14.49 & 0.902 & 1.58 \\
\hline$\sigma(a b s)$ & 0.103 & 0.041 & 0.021 & 0.030 & 5.218 & 0.055 & 0.155 & 0.358 & 0.371 & 0.008 & 0.126 \\
\hline $\mathrm{CV}$ & $1.9 \%$ & $0.6 \%$ & $8.5 \%$ & $3.3 \%$ & $18.8 \%$ & $7.9 \%$ & $3.3 \%$ & $2.2 \%$ & $2.6 \%$ & $0.9 \%$ & $8.0 \%$ \\
\hline
\end{tabular}

NOTE - The value of $K V$ for specimen 7 was lost because the optical encoder did not trigger.

\subsubsection{Calculation of sample size and outlier analysis}

The sample size calculated from HH-103 MCVN test results according to Eq. (1) is $n=1.4$ for KLST specimens and $n=2.4$ for RHS specimens ${ }^{*}$. For comparison, the values of sample size returned from the pilot lot testing of $\mathrm{HH}-103$ full-size specimens were 2.3 at $-40^{\circ} \mathrm{C}$ and 2.2 at room temperature.

The outlier analysis for $K V$ values performed by means of box-and-whiskers plots, shown in Figure 14 (KLST) and Figure 15 (RHS), detected one outlier among the RHS specimens (sample 6). No evidence of jamming or other atypical occurrences were associated to this specimen. However, the percentage of outliers ( $1 / 31$, or $3.2 \%$ ) is well below the $5 \%$ threshold for potential lot rejection [18].

"* The NIST procedure for standard Charpy specimens [18] uses the greater between $1.4 \mathrm{~J}$ and $5 \%$ of $K V$ in Eq. (1). The value $1.4 \mathrm{~J}$ was normalized by the ratio between $K V_{\text {full-size }}$ and $K V_{\text {sub-size }}$ for the specific energy level and MCVN type, i.e., 20.26 for KLST and 7.28 for RHS at the high-energy level (see section 5.2). 


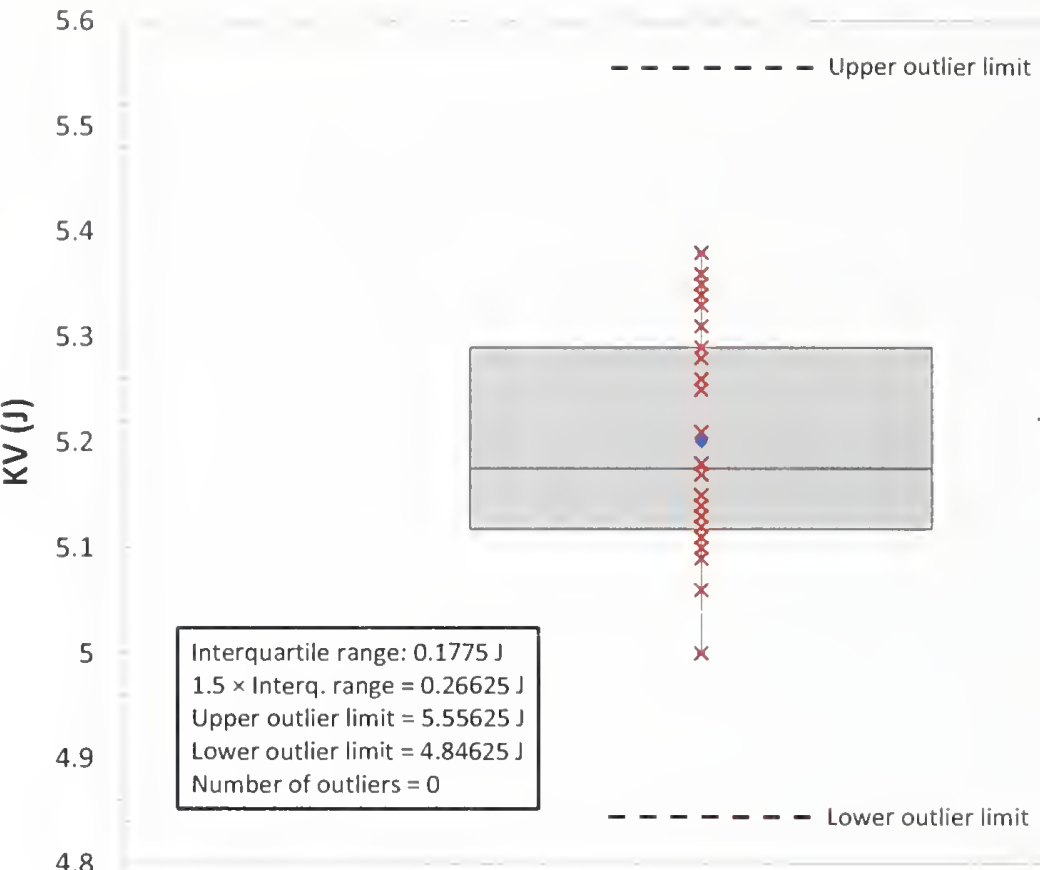

$\mathrm{HH}-103$

Figure 16 - Box-and-whisker plot for $K V$ values from KLST specimens of $\mathrm{HH}-103$.

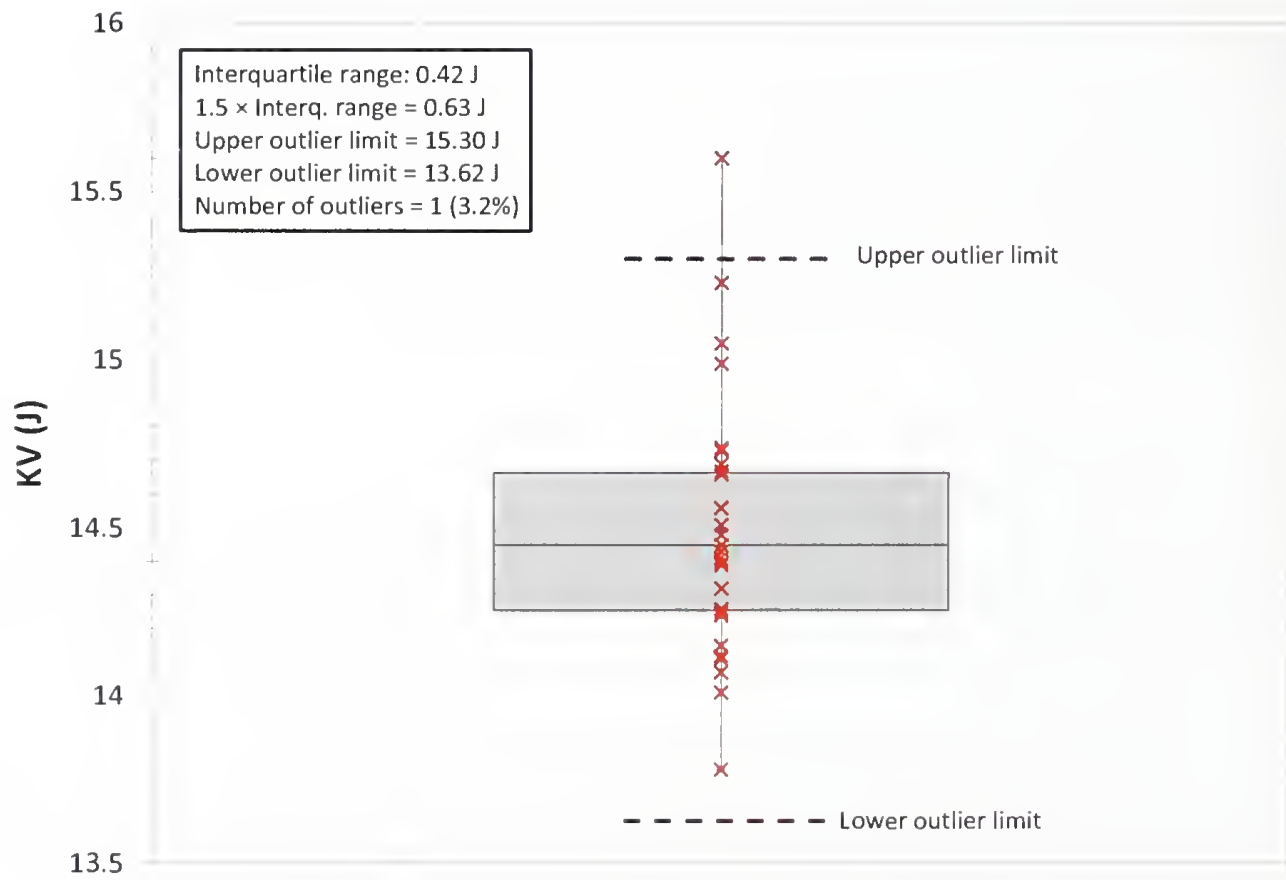

riH-103

Figure 17 - Box-and-whisker plot for $K V$ values from RHS specimens of $\mathrm{HH}-103$.

The results of the outlier analysis conducted by means of Grubbs' test are summarized in Table 9. 
Table 9 - Results of Grubbs' test for the detection of outliers on MCVN tests of HH-103.

\begin{tabular}{|c|c|c|c|c|}
\hline $\begin{array}{c}\text { Specimen } \\
\text { type }\end{array}$ & Parameter & $N$ & $\begin{array}{c}\text { Outliers } \\
\text { detected }\end{array}$ & $\begin{array}{c}\text { Outlier } \\
\text { specimens }^{\dagger \dagger}\end{array}$ \\
\hline & $F_{\mathrm{gy}}$ & 25 & 1 & 25 \\
& $F_{\mathrm{m}}$ & 25 & 0 & - \\
KLST & $W_{\mathrm{m}}$ & 25 & 0 & - \\
& $W_{\mathrm{t}}$ & 25 & 0 & - \\
& $K V$ & 32 & 0 & - \\
\hline \multirow{5}{*}{ RHS } & $F_{\mathrm{gy}}$ & 25 & 1 & 1 \\
& $F_{\mathrm{m}}$ & 25 & 0 & - \\
& $W_{\mathrm{m}}$ & 25 & 0 & - \\
& $W_{\mathrm{t}}$ & 25 & 0 & - \\
& $K V$ & 32 & 0 & - \\
\hline
\end{tabular}

Again, outliers were detected only for $F_{\mathrm{gy}}$ values. In this case, the outcome of Grubbs' test for $K V$ values from RHS tests does not coincide with the results of the box-and-whisker method. The outlier detected by the latter method (specimen 6, $K V=15.60 \mathrm{~J}$ ) is not classified as an outlier by Grubbs' test. For this test, the $\mathrm{Z}$ value is maximum (2.98), but remains below the critical value (3.25) at the $\alpha=0.01$ significance level. Note, however, that if Grubbs' test is performed at a significance level $\alpha=0.05$, the threshold value becomes 2.92 and specimen 6 is then also detected as an outlier.

\subsection{Super high-energy MCVN test results}

Only eight KLST specimens and eight RHS specimens from lot SH-36 were tested at room temperature. Additional tests are planned in the continuation of this project, so that an equivalent number of test results are available for each energy level and specimen configuration. Force, displacement and absorbed energy results are collected in Table 10 (KLST) and Table 11 (RHS), as well as average values, absolute standard deviations and percent coefficients of variation.

Table 10 - NIST test results from KLST specimens of SH-36 (super-high energy). Specimens 6-8 were tested non-instrumented.

\begin{tabular}{|c|c|c|c|c|c|c|c|c|c|c|c|}
\hline $\begin{array}{c}5 p e c i m e n \\
\text { id }\end{array}$ & $\begin{array}{c}F_{\mathrm{gV}} \\
(\mathrm{kN})\end{array}$ & $\begin{array}{c}F_{\mathrm{m}} \\
(\mathrm{kN})\end{array}$ & $\begin{array}{c}s_{\mathrm{gV}} \\
(\mathrm{mm})\end{array}$ & $\begin{array}{c}s_{\mathrm{m}} \\
(\mathrm{mm})\end{array}$ & $\begin{array}{c}s_{\mathrm{t}} \\
(\mathrm{mm})\end{array}$ & $\begin{array}{c}W_{\mathrm{gV}} \\
(\mathrm{J})\end{array}$ & $\begin{array}{c}W_{\mathrm{m}} \\
(\mathrm{J})\end{array}$ & $\begin{array}{c}W_{\mathrm{t}} \\
(\mathrm{J})\end{array}$ & $\begin{array}{c}K V \\
(\mathrm{~J})\end{array}$ & $K V / W_{\mathrm{t}}$ & $\begin{array}{c}W_{\mathrm{t}}-K V \\
(\mathrm{~J})\end{array}$ \\
\hline 1 & 1.66 & 1.96 & 0.36 & 1.40 & 11.10 & 0.31 & 2.25 & 10.89 & 9.77 & 0.897 & 1.12 \\
2 & 1.65 & 1.96 & 0.37 & 1.40 & 11.19 & 0.30 & 2.24 & 10.74 & 9.64 & 0.898 & 1.10 \\
3 & 1.65 & 1.97 & 0.33 & 1.40 & 11.34 & 0.27 & 2.27 & 10.84 & 9.72 & 0.897 & 1.12 \\
4 & 1.67 & 1.99 & 0.35 & 1.40 & 10.97 & 0.29 & 2.28 & 11.11 & 9.98 & 0.898 & 1.13 \\
5 & 1.65 & 1.96 & 0.33 & 1.38 & 11.04 & 0.28 & 2.24 & 10.66 & 9.60 & 0.901 & 1.06 \\
6 & & & & & & & & & 9.16 & & \\
7 & & & & & & & & & 9.57 & & \\
8 & & & & & & & & & 9.81 & & \\
\hline$N$ & 5 & 5 & 5 & 5 & 5 & 5 & 5 & 5 & 8 & 5 & 5 \\
Average & 1.66 & 1.97 & 0.35 & 1.40 & 11.13 & 0.29 & 2.26 & 10.85 & 9.66 & 0.898 & 1.11 \\
$\sigma$ (abs) & 0.009 & 0.013 & 0.018 & 0.009 & 0.143 & 0.016 & 0.018 & 0.171 & 0.240 & 0.002 & 0.028 \\
CV & $0.5 \%$ & $0.7 \%$ & $5.1 \%$ & $0.6 \%$ & $1.3 \%$ & $5.5 \%$ & $0.8 \%$ & $1.6 \%$ & $2.5 \%$ & $0.2 \%$ & $2.5 \%$ \\
\hline
\end{tabular}

${ }^{\dagger}$ See Table 7 (KLST) and Table 8 (RHS) for specimen numbers. 
Table 11 - NIST test results from RIS specimens of SH-36 (super-high energy). Specimens 6-8 were tested non-instrumented.

\begin{tabular}{|c|c|c|c|c|c|c|c|c|c|c|c|}
\hline $\begin{array}{c}\text { 5pecimen } \\
\text { id }\end{array}$ & $\begin{array}{l}F_{B Y} \\
(k N)\end{array}$ & $\begin{array}{l}F_{m} \\
(k N)\end{array}$ & $\begin{array}{c}s_{E Y} \\
(\mathrm{~mm})\end{array}$ & $\begin{array}{c}s_{m} \\
(\mathrm{~mm})\end{array}$ & $\begin{array}{c}s_{1} \\
(\mathrm{~mm})\end{array}$ & $\begin{array}{c}W_{g \gamma} \\
(J)\end{array}$ & $\begin{array}{l}W_{m} \\
\text { (J) }\end{array}$ & $\begin{array}{l}W_{t} \\
(J)\end{array}$ & $\begin{array}{l}K V \\
(J)\end{array}$ & $K V / W_{t}$ & $\begin{array}{c}W_{t}-K V \\
\text { (J) }\end{array}$ \\
\hline 1 & 5.20 & 6.83 & 0.28 & 1.23 & 11.28 & 0.69 & 6.79 & 30.38 & 28.31 & 0.932 & 2.07 \\
\hline 2 & 5.55 & 6.81 & 0.33 & 1.30 & 10.94 & 0.90 & 7.17 & 30.08 & 27.91 & 0.928 & 2.17 \\
\hline 3 & 5.53 & 6.81 & 0.41 & 1.29 & 11.06 & 1.00 & 6.71 & 29.56 & 27.34 & 0.925 & 2.22 \\
\hline 4 & 5.40 & 6.84 & 0.28 & 1.25 & 10.92 & 0.82 & 7.02 & 30.91 & 28.78 & 0.931 & 2.13 \\
\hline 5 & 5.37 & 6.82 & 0.28 & 1.21 & 10.97 & 0.82 & 6.74 & 29.88 & 27.66 & 0.926 & 2.22 \\
\hline 6 & & & & & & & & & 30.17 & & \\
\hline 7 & & & & & & & & & 30.05 & & \\
\hline 8 & & & & & & & & & 30.07 & & \\
\hline$N$ & 5 & 5 & 5 & 5 & 5 & 5 & 5 & 5 & 8 & 5 & 5 \\
\hline Average & 5.41 & 6.82 & 0.32 & 1.26 & 11.03 & 0.85 & 6.89 & 30.16 & 28.79 & 0.928 & 2.16 \\
\hline$\sigma(a b s)$ & 0.141 & 0.013 & 0.057 & 0.038 & 0.148 & 0.114 & 0.200 & 0.514 & 1.166 & 0.003 & 0.064 \\
\hline $\mathrm{CV}$ & $2.6 \%$ & $0.2 \%$ & $18.0 \%$ & $3.1 \%$ & $1.3 \%$ & $13.5 \%$ & $2.9 \%$ & $1.7 \%$ & $4.1 \%$ & $0.3 \%$ & $3.0 \%$ \\
\hline
\end{tabular}

With respect to the scatter of CVN test results documented in [17], the cocfficients of variation for $K V_{\mathrm{MCVN}}$ are similar (KLST) or slightly higher $\left(\right.$ RHS $^{\ddagger \dagger}$ ): we obtained $2.5 \%$ and $4.1 \%$, respectively, compared with $\mathrm{CV}=$ $2.5 \%$ from CVN specimens. Values of absorbed energy $W_{\mathrm{t}}$ calculated from the instrumented test records provide slightly lower values of CV (1.6\% for KLST and $1.7 \%$ for RHS) than for CVN tests $(2.18 \%)$. As far as force values are concerned, the scatter of $C V N$ results from [17] $\left(C V_{\mathrm{Fgy}}=1.3 \%\right.$ and $\left.C V_{\mathrm{Fm}}=0.4 \%\right)$ is intermediate between the coefficients of variations calculated for KLST and RHS specimens.

We did not calculate sample sizes or perform outlier detection, given the limited number of super-high energy tests performed. These analyses will be done once additional MCVN test results are available.

\footnotetext{
㧊 Oddly enough, the three non-instrumented tests (sec Table 11) yielded $K V$ values greater than $30 \mathrm{~J}$, clearly higher than those measured from the five instrumented tests, which ranged between $27.34 \mathrm{~J}$ and $28.31 \mathrm{~J}$. The reason for this is unclear, but it obviously affects the value of the coefficient of variation (if non-instrumented test are ignored, we obtain $\mathrm{CV}=2.0 \%$, i.e, lower than for CVN specimens).
} 


\section{Additional aspects investigated}

\section{$5.1 \quad$ Comparison between different measures of absorbed energy ( $W_{\mathrm{t}}$ and $\left.K V\right)$}

The result tables for all energy levels and MCVN specimen types also provide the ratio and the difference between instrumented total energy $W_{1}$ and encoder absorbed energy $K V$ ior each instrumented test pcrformcd. Typically, the relationship between $W_{\mathrm{t}}$ and $K V$ varies from machinc to machine and depends on the characteristics of the instrumented striker, such as striker configuration $(2 \mathrm{~mm}$ vs. $8 \mathrm{~mm}$ ), position of strain gages, striker calibration, etc. [20,21].

At the high and super-high energy levels, the ratio $K V / W_{\mathrm{t}}$ is extremely consistent for both KLST and RHS tests, with coefficients of variations $\mathrm{CV}<1 \%$ (see Table 7 and Table 8 (high) and Table 10 and Table 11 (super-high)). At both energy levels, absolute values of $K V / W_{\mathrm{t}}$ are also similar for the two configurations ( 0.9). At the low-energy level, more variability is observed, particularly for RHS specimens ( $\mathrm{CV}=3.6 \%$ ). For KLST specimens, $\mathrm{CV}$ is just above $1 \%(1.3 \%)$. Absolute values for $K V / W_{\mathrm{t}}$ are again relatively close for the two specimen types: 1.058 for KLST and 0.984 for RHS.

The average differences between $K V$ and $W_{\mathrm{t}}$ are lower than $1 \mathrm{~J}$ at the low-energy level, less than $2 \mathrm{~J}$ at the high-energy level, and slightly higher than $2 \mathrm{~J}$ at the super-high energy level. Small differences between encoder and instrumented absorbed energies typically indicate a satisfactory performance of impact machine [20,21].

\subsection{Correlation between full-size and miniaturized specimen absorbed energies}

An extensive overview of the existing correlations of upper shelf energy (USE) data between full-size and miniaturized Charpy specimens was provided by Sokolov and Alexander [22]. An additional correlation approach was presented by the author [23].

The method commonly used in Europe consists of establishing an cmpirical ratio between full-size USE $\left(U S E_{f s}\right)$ and sub-size $U S E$ (USE $E_{s s}$ ) based on a large number of tests. A different approach, often used by North American and Japanese researchers, correlates USE values with the ratio of various geometrical parameters, $G P_{x}$ (with $x=f s$ or $s s$ ) for different specimen geometries in the form:

$$
\frac{U S E_{f s}}{U S E_{s s}}=\frac{G P_{f s}}{G P_{s S}}
$$

Eq. (2) can also be expressed in terms of a normalization factor $N F$, which is precisely the ratio of geometrical parameters mentioned above:

$$
U S E_{f s}=N F \times U S E_{s s}
$$

The most common expressions for NF that can be found in the literature are the following:

$$
N F_{1}=\frac{\beta b f_{s}}{\beta b},
$$

based on the ratio of fracture areas, with $B=$ specimen thickness and $b=$ ligament size $[24,25]$; 


$$
\left.N F_{2}=-\frac{\left[3 b^{3 / 2}\right.}{3 b^{3 / 2}}\right]
$$

based on the ratio of nominal fracturc volumes $[24,25]$;

$$
N F_{3}=\frac{\left(\beta b^{2}\right)}{\left(k b^{2}\right)_{s}^{+}},
$$

bascd on a different cxpression for the ratio of nominal fracturc volumes [26,27], and

$$
N F_{4}=\frac{\left(\frac{B b^{2}}{L K_{t}}\right)_{f s}}{\left(\frac{B b^{2}}{L K_{t}}\right)_{s S}}
$$

where $L=$ span and $K_{\mathrm{t}}=$ elastic stress concentration factor [28].

In addition, Sokolov and Alexander [22] established empirical values $N F_{5}$ for 4 types of sub-size specimens considered in their study ${ }^{\S \S}$ by avcraging the values of $U S E_{f s} / U S E_{S S}$ obtained on ten diffcrent materials (mostly pressure vessel stcels with different heat treatments).

In [23], an exponcntial relationship between full-size and KLST values of USE was established, as shown in Figure 18. The best-fit regression curve relating $U S E_{f s}$ and $U S E_{K L S T}$ is given by

$$
U S E_{f S}=29.454 e^{0.2378 U S E_{K L S T}} \quad .
$$

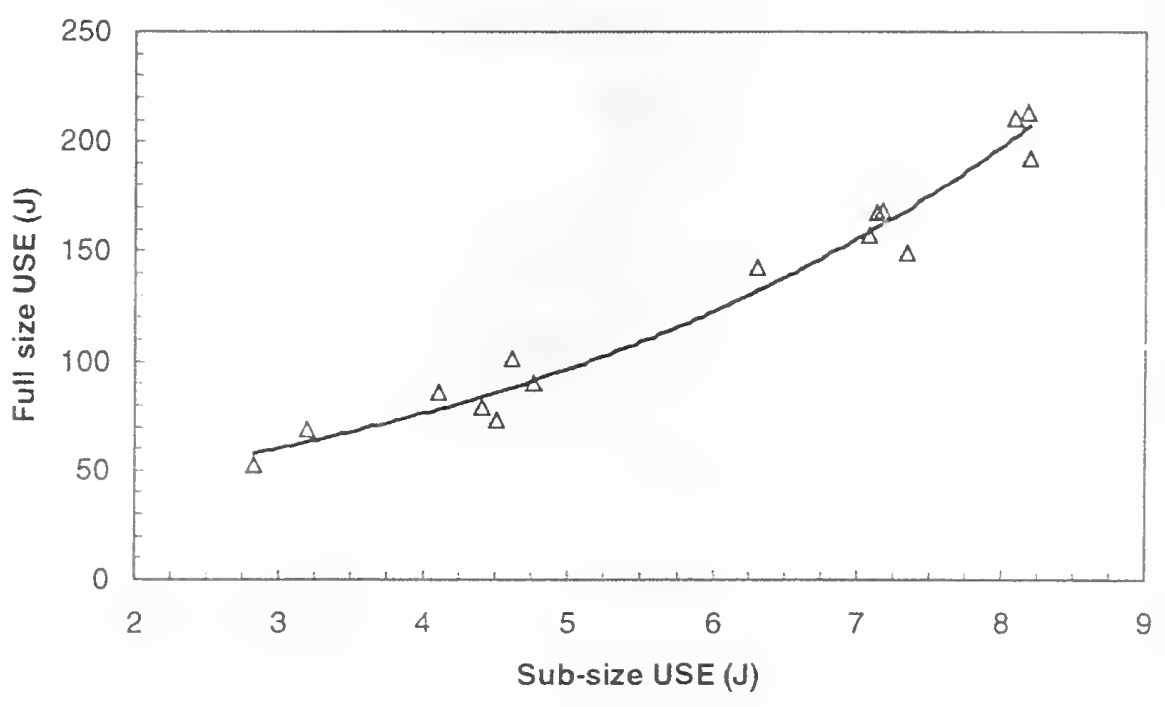

Figure 18 - Exponential correlation between sub-size and full-size USE values for KLST specimens [23].

\footnotetext{
$\$$ In [22], sub-size specimen type 4 corresponds to KLST; sub-size specimen type 3 (cioss section $5 \mathrm{~mm} \times 5 \mathrm{~mm}$, length 27 $\mathrm{mm}$, span $20 \mathrm{~mm}$ ) is dimensionally almost identical to RHS.
} 
Finally, the average value of $U S E_{\mathrm{fs}} / U S E_{\mathrm{KLST}}$ calculated in [23] for nine unirradiated and irradiated pressure vessel steels was $N F_{7}=21.6$.

A summary of the different normalization factors available for the miniaturized specimen geometries considered in this study (KLST and RHS), according to the methods listed above, is provided in Table 12.

Table 12 - Summary of normalization factors for estimating full-size $U S E$ based on miniaturized $U S E$, see eq. (3). Note: the value of $N F_{6}[23]$ is not shown in the Table, because it depends on $U S E_{\mathrm{fs}}$.

\begin{tabular}{|c|c|c|c|c|c|c|}
\hline $\begin{array}{c}\text { Specimen } \\
\text { type }\end{array}$ & $\begin{array}{c}N F_{1} \\
\text { [Eq.(4)] }\end{array}$ & $\begin{array}{c}N F_{2} \\
\text { [Eq.(5)] }\end{array}$ & $\begin{array}{c}N F_{3} \\
\text { [Eq.(6)] }\end{array}$ & $\begin{array}{c}N F_{4} \\
\text { [Eq.(7)] }\end{array}$ & $\begin{array}{c}N F_{5} \\
{[22]}\end{array}$ & $\begin{array}{c}N F_{7} \\
{[23]}\end{array}$ \\
\hline KLST & 8.9 & 26.5 & 23.7 & 13 & 24.9 & 21.6 \\
\hline RHS & 4.3 & 8.9 & 8.9 & $2.8^{* * *}$ & $6.8^{\S \S}$ & N/A \\
\hline
\end{tabular}

Considering the MCVN tests performed at three energy levels on KLST and RHS specimens, experimental normalization factors $N F_{\text {exp }}$ were calculated by dividing the certified/average values of $K V_{f s}$ at room temperature by the average MCVN absorbed energies for every data set. The results are shown in Table 13 and should be compared with the normalized factors listed in Table 12.

Table 13 - Experimental normalization factors obtained from NIST MVCN tests.

\begin{tabular}{|c|c|c|c|}
\hline $\begin{array}{l}\text { Energy } \\
\text { level }\end{array}$ & $\begin{array}{l}\text { Specimen } \\
\text { type }\end{array}$ & $\begin{array}{l}\overline{K V} \\
(\mathrm{~J})\end{array}$ & $N F_{\text {exp }}=\frac{\overline{K V}_{f s}}{\overline{K V}_{s s}}$ \\
\hline Low & $\begin{array}{c}\text { Full-size } \\
\text { KLST } \\
\text { RHS }\end{array}$ & $\begin{array}{l}18.2 \\
1.4 \\
3.4\end{array}$ & $\begin{array}{c}- \\
13.3 \\
5.3\end{array}$ \\
\hline High & $\begin{array}{c}\text { Full-size } \\
\text { KLST } \\
\text { RHS }\end{array}$ & $\begin{array}{c}105.3 \\
5.2 \\
14.5\end{array}$ & $\begin{array}{c}- \\
20.2 \\
7.3\end{array}$ \\
\hline Super-high & $\begin{array}{c}\text { Full-size } \\
\text { KLST } \\
\text { RHS }\end{array}$ & $\begin{array}{c}239.8 \\
9.7 \\
28.8\end{array}$ & $\begin{array}{c}- \\
24.8 \\
8.3\end{array}$ \\
\hline
\end{tabular}

Theoretical (Table 12), empirical (Figure 18) and experimental (Table 13) normalization factors are compared in Figure 19 for KLST tests and in Figure 20 for RHS tests.

${ }^{* * *}$ Values obtained for sub-size specimen type 3 (almost identical to RHS). 


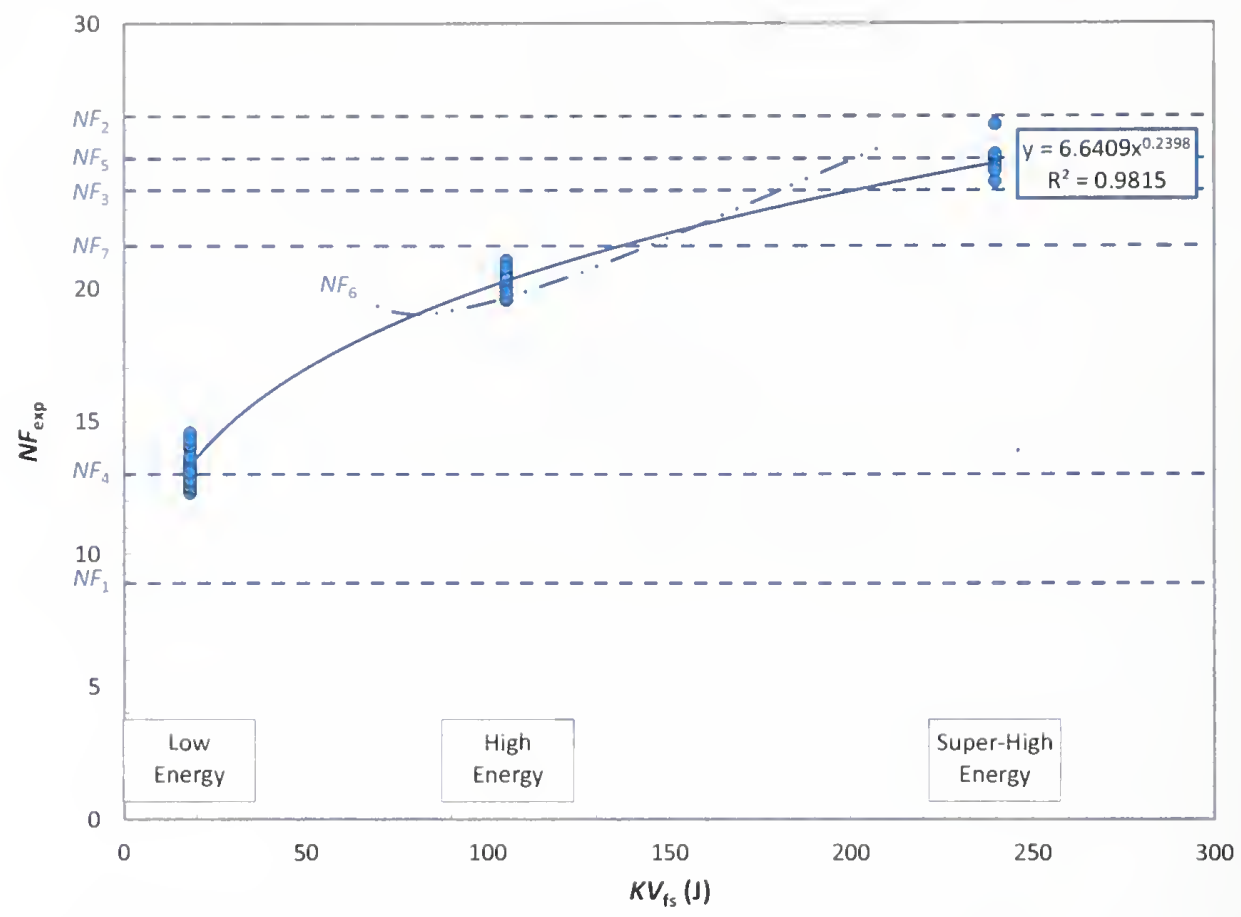

Figure 19 - Comparison between theoretical, empirical and experimental normalization factors for KLST specimens of different energy levels.

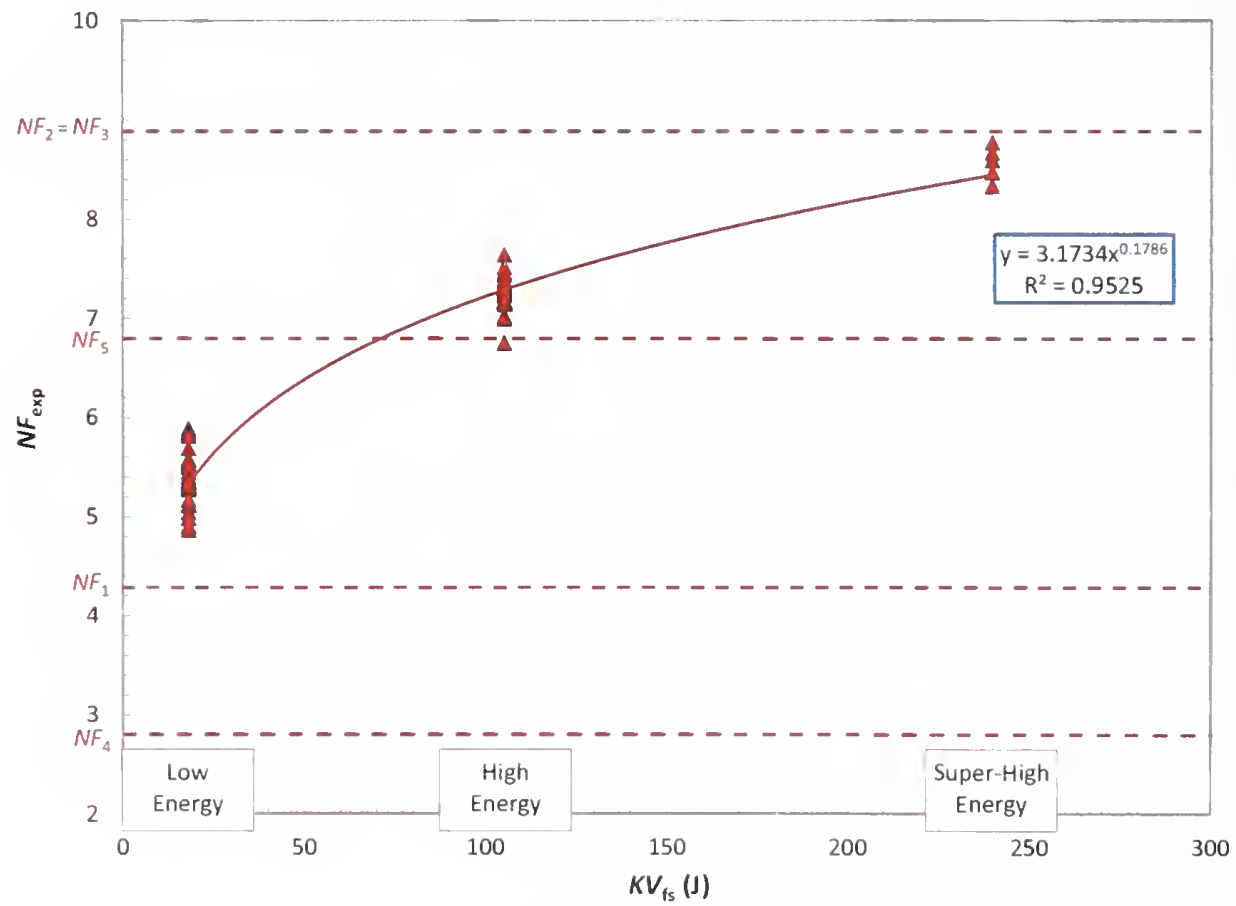

Figure 20 - Comparison between theoretical and experimental normalization factors for RHS specimens of different energy levels. 
Another approach for evaluating the different normalization factors is shown in Figure 21 (KLST) and Figure 22 (RHS), where the certified or average values of $K V_{f s}$ are compared with predictions obtained from $K V_{s s}$ by the use of the various methods presented.

First of all, it must be stated that all the methods described above address USE values, rather than generic values of absorbed energy. At the high and super-high energy levels, analysis of the instrumented traces indicates fully ductile behavior; therefore it is legitimate to assume $K V=U S E$. For low-energy specimens, however, the material's behavior is typical of the ductile-to-brittle transition regime (shear fracture appearance values, estimated through the analysis of the instrumented test record, ranged between $40 \%$ and $60 \%$ for KLST specimens and between $30 \%$ and $50 \%$ for RHS specimens).

The most immediate conclusion emerging from Figure 19 and Figure 20 is that normalization factors are not independent of absorbed energy. Furthermore, full-size $K V$ values estimated by means of normalization factors $N F_{1}$ and $N F_{4}$ are acceptable only at the low energy level, while at higher energies they are severely underpredicted. Conversely, non-conservative estimations were generally obtained through the use of $N F_{2}$. Mixed results are observed for $N F_{3}, N F_{5}$ and $N F_{6}$. Equation (8) works only for KLST tests at the high-energy level, i.e., when both $K V_{\text {fs }}$ and $K V_{\text {KLST }}$ are within the ranges for which the relationship was developed (Figure 18). In short, none of the approaches considered appears convincing for all energy levels and both MCVN miniaturized specimen types.

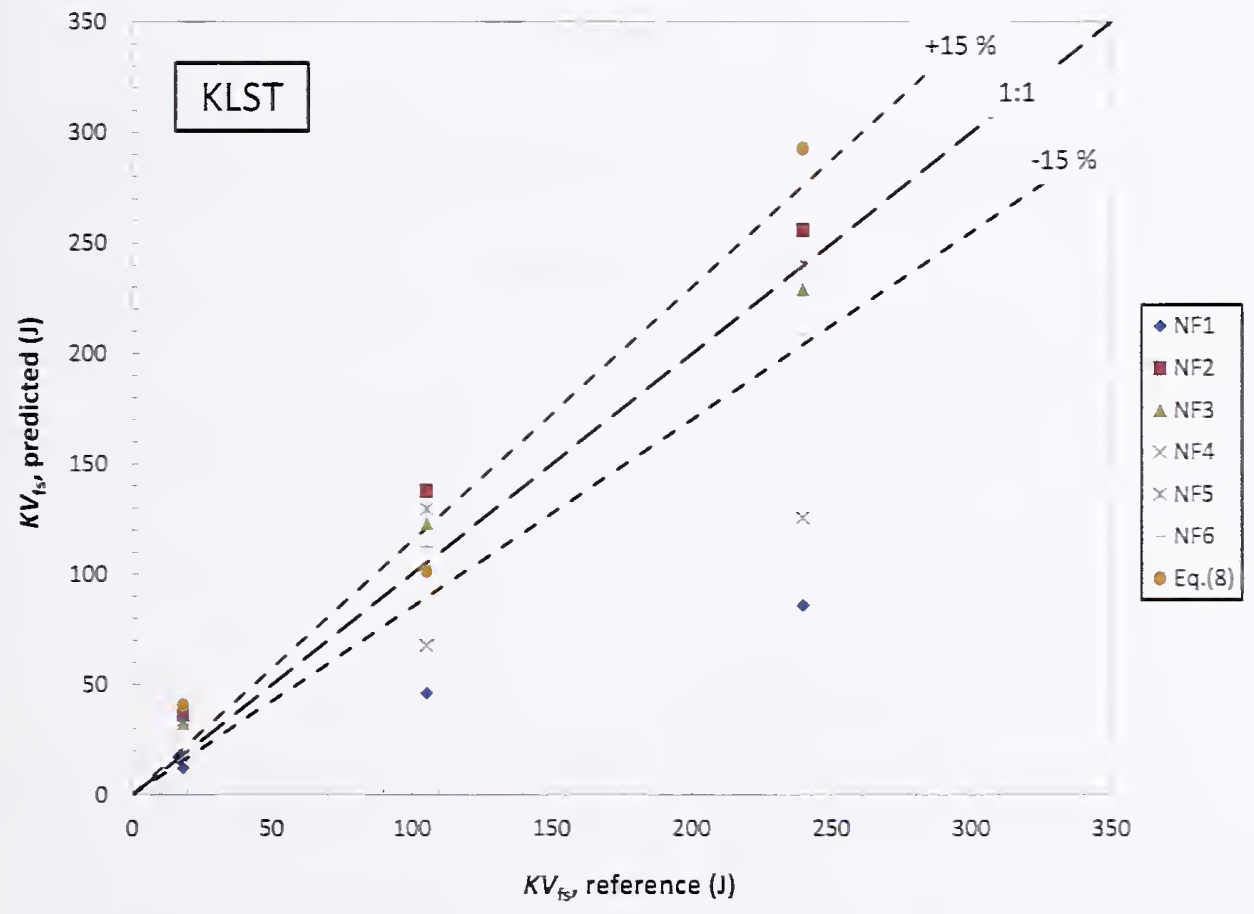

Figure 21 - Prediction of full-size absorbed energy from KLST specimens by use of different approaches. 


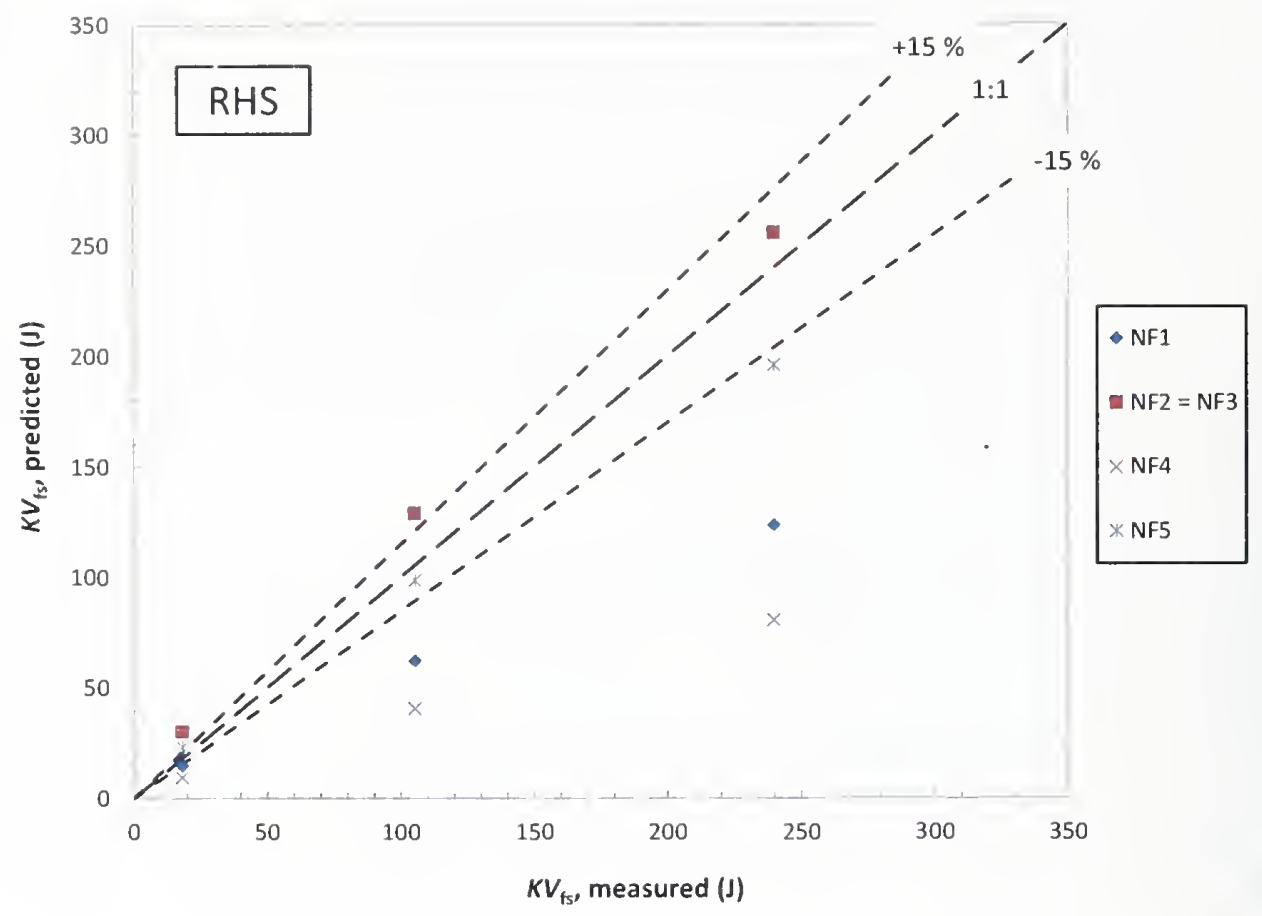

Figure 22 - Prediction of full-size absorbed energy from RHS specimens with different approaches.

Based on our test results, the following relationships between $K V_{s s}$ and $K V_{f s}$ were obtained:

$$
K V_{f s}=12102 \cdot K V_{K L S T}^{1.313}
$$

for KLST specimens (with coefficient of determination $R^{2}=0.9981$ ), and

$$
K V_{f s}=4.1397 \cdot K V_{R H S}^{1.2091}
$$

for RHS specimens (with $R^{2}=0.9978$ ).

\subsection{Symmetrical versus asymmetrical fracture}

When a Charpy specimen fractures, shear lips are formed unless the material is fully brittle (i.e., shear fracture appearance $=0 \%$ ). Shear lips are jagged edges generated at the lateral borders of the fracture surface. Their magnitude (height) is directly related to amount of ductility exhibited by the Charpy specimen at the test temperature. When shear lips are visible on a tested Charpy specimen, the fracture is defined symmetrical if both shear lips are on the same specimen half, and asymmetrical if each specimen half exhibits one shear lip (Figure 23). 


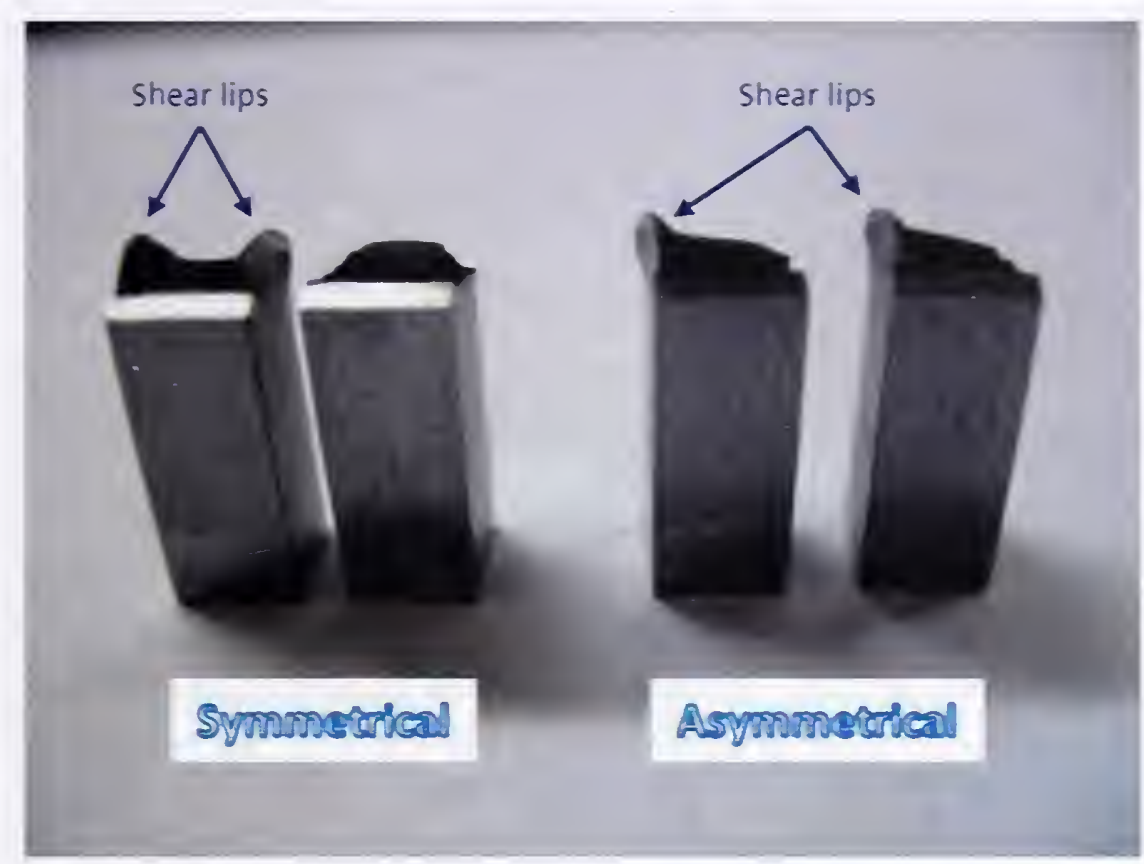

Figure 23 - Examples of symmetrical and asymmetrical fracture on high-energy RHS specimens.

Earlier investigations at NISI [29,30] conducted on full-size verification specimens of high and super-high energy showed that the symmetry of shear lip formation has a significant influence on absorbed energy. Specifically, asymmetrical fractures are generally associated with higher absorbed energies.

For the MCVN specimens tested at NIST for this study. the information on fracture symmetr. is summarized in Table 14.

Table 14 - Symmetrical (S) and asymmetrical (A) fractures for MCVN tests performed at NIST.

\begin{tabular}{|c|c|c|c|c|c|}
\hline $\begin{array}{l}\text { Energy } \\
\text { level }\end{array}$ & $\begin{array}{c}\text { Specimen } \\
\text { type }\end{array}$ & $\begin{array}{l}\text { Type of } \\
\text { fracture }\end{array}$ & $\begin{array}{l}\text { No. of } \\
\text { tests }\end{array}$ & $\begin{array}{l}\overline{K T^{\prime}} \\
(J)\end{array}$ & $\begin{array}{c}\sigma_{K \overline{K L}} \\
(\mathrm{~J})\end{array}$ \\
\hline \multirow{4}{*}{ Low } & \multirow{2}{*}{ KLST } & $\mathrm{S}$ & $18(56 \%)$ & 1.37 & $0.0 \div 6$ \\
\hline & & $A$ & $1 \div\left(1+\frac{1}{2} \%\right)$ & 1.36 & 0.078 \\
\hline & \multirow[b]{2}{*}{ RHS } & $\mathrm{S}$ & $16(50 \%)$ & 3.47 & 0.127 \\
\hline & & $A$ & $16(50 \%)$ & 3.38 & 0.198 \\
\hline \multirow{4}{*}{ High } & \multirow{2}{*}{ KLST } & $S$ & $12(38 \%)$ & 5.12 & 0.062 \\
\hline & & $A$ & $20(63 \%)$ & 5.25 & 0.091 \\
\hline & \multirow{2}{*}{ RHS } & $S$ & $14(+1 \%)$ & 14.28 & 0.239 \\
\hline & & $A$ & $18(56 \%)$ & 14.67 & 0.373 \\
\hline \multirow{4}{*}{ Supar-High } & \multirow{2}{*}{ KLST } & $S$ & $6(75 \%)$ & 9.58 & 0.217 \\
\hline & & A & $2(25 \%)$ & 9.90 & 0.120 \\
\hline & \multirow{2}{*}{ RHS } & $\mathrm{S}$ & $8(100 \%)$ & 28.79 & 1.166 \\
\hline & & $A$ & $0(0 \%)$ & - & - \\
\hline
\end{tabular}

The likelihood of symmetrical or asymmetrical fracture appears close at low and high energies. while at the super-high energy level, symmetrical fracture clearly prevails. In order to statistically assess the influence of fracture symmetry on absorbed energy. Student's $t$-test [31] was applied to the means of $K T$ for specimens fracturing symmetrically and asymmetrically for each MCV configuration and nominal energy- level. 
The specific $t$-test uscd is the unpaired two-sample location test, which verifies the null hypothesis that the means of two normally distributed populations are equal. The results obtaincd are summarized in Table 15.

Table 15 - Results of Student's $t$-test for MCVN tests performed at NIST.

\begin{tabular}{|c|c|c|c|c|c|c|c|}
\hline $\begin{array}{l}\text { Energy } \\
\text { level }\end{array}$ & $\begin{array}{l}\text { Specimen } \\
\text { type }\end{array}$ & $\begin{array}{l}\text { Type of } \\
\text { fracture }\end{array}$ & $\begin{array}{l}\overline{K V} \\
(\mathrm{~J})\end{array}$ & $\begin{array}{l}\text { Two-tailed } \\
P \text { value }\end{array}$ & $t$ & $\begin{array}{l}\text { Standard error } \\
\text { of difference }\end{array}$ & Test result \\
\hline \multirow{2}{*}{ Low } & KLST & $\begin{array}{l}\mathrm{S} \\
\mathrm{A}\end{array}$ & $\begin{array}{l}1.37 \\
1.36\end{array}$ & 0.8327 & 0.2080 & 0.023 & $\begin{array}{c}\text { Means are not } \\
\text { statistically different }\end{array}$ \\
\hline & RHS & $\begin{array}{l}\text { S } \\
\text { A }\end{array}$ & $\begin{array}{l}3.47 \\
3.38 \\
\end{array}$ & 0.1213 & 1.5945 & 0.059 & $\begin{array}{l}\text { Means are not } \\
\text { statistically different }\end{array}$ \\
\hline \multirow{2}{*}{ High } & KLST & $\begin{array}{l}\mathrm{S} \\
\mathrm{A}\end{array}$ & $\begin{array}{l}5.12 \\
5.25\end{array}$ & 0.0001 & 4.4804 & 0.030 & $\begin{array}{l}\text { Means are statistically } \\
\text { extremely different }\end{array}$ \\
\hline & RHS & $\begin{array}{l}\mathrm{S} \\
\mathrm{A}\end{array}$ & $\begin{array}{l}14.28 \\
14.67\end{array}$ & 0.0022 & 3.3579 & 0.116 & $\begin{array}{c}\text { Means are statistically } \\
\text { very different }\end{array}$ \\
\hline \multirow{2}{*}{ Super-High } & KLST & $\begin{array}{l}\mathrm{S} \\
\mathrm{A}\end{array}$ & $\begin{array}{l}9.58 \\
9.90\end{array}$ & 0.1050 & 1.9078 & 0.167 & $\begin{array}{c}\text { Means are not } \\
\text { statistically different }\end{array}$ \\
\hline & RHS & $\begin{array}{l}\mathrm{S} \\
\mathrm{A}\end{array}$ & $\begin{array}{c}28.79 \\
-\end{array}$ & - & - & - & - \\
\hline
\end{tabular}

NOTE - The $P$ value can be interpreted as the probability of the difference between sample means being coincidental.

The results of the statistical analyses show that fracture symmetry has no significant influence at low-energy level, whereas at high-encrgy level, asymmetric fracture is associated with higher absorbed energies. Results at super-high level (only KLST specimens) are not reliable due to the limited sample sizes (only twoasymmetrical fractures). These results are consistent with the findings reported in $[29,30]$, where only high and super-high verification specimens were analyzed.

\subsection{Broken versus unbroken specimens}

Another characteristic feature of Charpy testing is the fact that, after being struck by the hammer, specimens may exit the anvils either broken in two pieces or unbroken. The latter happens when the material is ductile enough to be bent and pushed out through the anvils by the striker before being fully fractured. In some cases, tested specimens are still in one piece, but the remaining ligament is so thin that samples can be easily broken by applying moderate force with one's fingers. In this case, the specimen is classified as "finger broken," and for practical purposes is considered equivalent to "broken." "ौ+† Note also that, according to ASTM E23-07 $7^{\varepsilon 1}$, for unbroken specimens, the percent shear fracture is conventionally given as $100 \%$.

Chandavale and Dutta [32] investigated the amount of impact energy spent for tossing the specimen. This toss cnergy is considered an crror in the absorbed energy value returned by the machine encoder, which should be subtracted from $K V$ in order to obtain the real fracture energy. A combination of theoretical calculations and expcrimcnts showed that, for a low-carbon steel (ASTM A516 Gr. 70) tested at room temperature on a 300-J pendulum and providing $K V \approx 100 \mathrm{~J}$, this error is on the order of $1.25 \mathrm{~J}$. IF the average ratio between $\mathrm{CVN}$ and

\footnotetext{
${ }^{++\dagger}$ Section 9.2.2 of ASTM E23-0 $7^{\varepsilon l}$ reads: "The lateral expansion of an unbroken specimen can be reported as broken if the specimen can be separated by pushing the hinged halves together once and then pulling them apart without further fatiguing the specimen (...)".
} 
MCVN absorbed energies calculated from high-energy NIST tests (see section 5.2) is used, this error would correspond to $0.06 \mathrm{~J}$ for KLST and $0.17 \mathrm{~J}$ for RHS.

In the case of miniaturized Charpy specimens. the reduced dimensions enhance material ductility and thus increase the likelihood of specimens exiting the pendulum unbroken. This is particularly true for KLSI specimens, which are more slender than RHS and have a larger span value (22 mm instead of $19.3 \mathrm{~mm}$ ).

The information concerning the percentage of broken and unbroken MCVN specimens from NIST tests is provided in Table 16.

Table 16 - Percentages of broken (B), finger-broken (FB) and unbroken (U) specimens for the MCVN tests performed at NIST.

\begin{tabular}{|c|c|c|c|c|c|}
\hline $\begin{array}{c}\text { Energy } \\
\text { level }\end{array}$ & $\begin{array}{c}\text { Specimen } \\
\text { type }\end{array}$ & $\begin{array}{c}\text { No. of } \\
\text { tests }\end{array}$ & B & FB & U \\
\hline \multirow{4}{*}{ Low } & KLST & 32 & $\begin{array}{c}32 \\
(100 \%)\end{array}$ & $\begin{array}{c}0 \\
(0 \%)\end{array}$ & $\begin{array}{c}0 \\
(0 \%)\end{array}$ \\
\cline { 2 - 6 } & RHS & 32 & $\begin{array}{c}32 \\
(100 \%)\end{array}$ & $\begin{array}{c}0 \\
(0 \%)\end{array}$ & $\begin{array}{c}0 \\
(0 \%)\end{array}$ \\
\hline \multirow{4}{*}{ High } & KLST & 32 & $\begin{array}{c}0 \\
(0 \%)\end{array}$ & $\begin{array}{c}14 \\
(44 \%)\end{array}$ & $\begin{array}{c}18 \\
(56 \%)\end{array}$ \\
\cline { 2 - 6 } & RHS & 32 & $\begin{array}{c}7 \\
(22 \%)\end{array}$ & $\begin{array}{c}19 \\
(59 \%)\end{array}$ & $\begin{array}{c}6 \\
(19 \%)\end{array}$ \\
\hline \multirow{4}{*}{ Super-High } & KLST & 8 & $\begin{array}{c}0 \\
(0 \%)\end{array}$ & $\begin{array}{c}0 \\
(0 \%)\end{array}$ & $\begin{array}{c}8 \\
(100 \%)\end{array}$ \\
\cline { 2 - 6 } & RHS & 8 & $\begin{array}{c}0 \\
(0 \%)\end{array}$ & $\begin{array}{c}0 \\
(0 \%)\end{array}$ & $\begin{array}{c}8 \\
(100 \%)\end{array}$ \\
\hline
\end{tabular}

At the low and super-high energy level, all MCVN specimens tested exit the anvils broken or unbroken. respectively. For high-energy specimens, however, constraint conditions can vary from specimen to specimen, and specimens can be broken, finger-broken or unbroken. At this energy level, a clear difference can be observed between KLST and RHS, with the former specimen type providing clearly more unbroken tests ( $56 \%$ ) than the latter (19\%). Moreover, none of the tested KLST specimen exited the anvils in two pieces (i.e., B $=0 \%$ ). As previously mentioned, different specimen proportions and anvil spacing are the causes for these differences in behavior.

For high-energy KLST specimens, an unpaired two-tailed $t$-test detected a statistically extremely significant difference $(P$-value $<0.0001)$ between the mean $K V$ of finger-broken $(5.12 \mathrm{~J})$ and unbroken $(5.26 \mathrm{~J})$ specimens. Note also that all the unbroken specimens exhibited asymmetrical fracture, while the percentage of symmetrical and asymmetrical fractures among the finger-broken specimens was $86 \%$ and $14 \%$, respectively. In the case of RHS specimens. the $t$-test failed to detect a clear statistical significant difference $(P$-value $=0.0610)$ between the mean $K V$ of broken/finger-broken $(14.44 \mathrm{~J})$ and unbroken $(14.78 \mathrm{~J})$ specimens $s^{\frac{+*}{*}}$. For broken/finger-broken RHS specimens, fractures are nearly equally split between symmetrical (54\%) and asymmetrical (46\%); all six unbroken specimens exhibited asymmetrical fracture.

This is probably due to the small sample size of the unbroken specimens $(6 \%)$. 


\section{Conclusions}

This report describes the experimental results obtained at NIST by testing miniaturized Charpy speeimens of two different eonfigurations (KLST and RHS) at three energy levels (low, high and super-high). These tests eonstitute the preliminary qualifieation phase of a NIST project aimed at producing a new SRM, i.e., miniaturized referenee speeimens for the indirect verification of small-seale impaet testing maehines.

The most important eonelusions for the NIST projeet are the following:

1. The new small-seale instrumented impaet tester purehased by NIST was suceessfully qualified by testing KLST speeimens of the JRQ pressure vessel steel and eomparing the results with data from an international Round-Robin condueted by a European technieal eommittee in the mid-90s. Exeellent agreement between NIST and Round-Robin data was observed.

2. The results obtained for absorbed energy $K V$ from MCVN speeimens at low and high energy indieate that production and eertifieation of a new SRM may be feasible for the verification of both absorbed energy and maximum force. Coefficients of variation and sample sizes for MCVN specimens were slightly higher than for full-size specimens at low energy, and very similar at high energy. In general, result variability was smaller for KLST than for RHS. For maximum forees, the eoeffieients of variations from NIST tests, whieh range from $0.6 \%$ to $1.8 \%$, are smaller than those reported for the Round-Robin that qualified the dynamie force verification speeimens from the same batehes ( $2.3 \%$ for low energy and $1.2 \%$ for high energy). Outlier analysis was performed with box-and-whiskers plots and Grubbs' tests. For both low-energy and high-energy tests, outlier values were deteeted by Grubbs' test for forees at general yield. One RHS high-energy speeimen was also elassified as an outlier by the box-and-whiskers method but not by Grubbs' test at the $\alpha=0.01$ signifieanee level.

3. At the super-high energy level, a limited number of MCVN speeimens (eight) were tested. The aim of these tests was to determine whether absorbed energy values would differ signifieantly from those obtained at the high-energy level. The answer is clearly affirmative: for KLST and RHS speeimens, we obtained average values $K V=9.66 \mathrm{~J}$ and $28.79 \mathrm{~J}$, respectively. For eomparison, average KLST and RHS values at the high-energy level were $K V=5.20 \mathrm{~J}$ and $14.49 \mathrm{~J}$. This justifies the eontinued development of super-high energy MCVN verifieation speeimens. Additional tests on super-high energy MCVN speeimens will be performed in the eontinuation of this projeet.

Additional researeh aspeets were also investigated in this preliminary phase of the projeet. The most signifieant eonelusions are summarized here:

(a) For the NIST instrumented small-scale impact tester, the relationship between absorbed energies returned by the eneoder $(K V)$ and ealeulated from the test reeord $\left(W_{1}\right)$ is quite eonsistent. The ratio $K V / W_{1} \approx 1.0$ at low energy and $K V / W_{1} \approx 0.9$ at high and super-high energy. The performanee of the maehine ean be considered satisfactory.

(b) Average energy values from MCVN speeimens were correlated to eertified/average energy values for full-size speeimens at the eorresponding energy levels. The results were compared to normalization approaehes published in the literature. It is elear that normalization faetors $\left(K V_{C V N} / K V_{M C V N}\right)$ are not eonstant. but depend on the energy level and possibly also material's strength. It was found that none of the published methods was 
fully satisfactory at all energy levels. Empirical correlations were obtained for KLST and RHS specimens by fitting the data with power-law curves.

(c) We investigated the relationship between fracture symmetry (i.e., both shear lips on the same specimen half or one on either half) and absorbed energy, as well as the relative frequency of symmetrical and asymmetrical fracture at the different energy level. At low energies, both types of fracture appear equally likely, but no influence was detected on absorbed energy by use of statistical tools (Student's $t$-test). At high and super-high energies, asymmetrical fracture is more likely to occur and was found to be associated with higher absorbed energies. This is consistent with a previous study conducted at NIST on full-size verification specimens.

(d) Another feature we looked at is whether specimens are fully broken, "finger-broken" or unbroken after being tested. At low and super-high energy levels, respectively, all specimens exit the anvils fully broken and unbroken. For the high-energy specimens, a mix of broken/finger broken and unbroken specimens was observed, with KLST specimens showing a more pronounced tendency to exit the anvils unbroken. Unbroken samples are also associated with a statistically higher absorbed energy.

In the next phase of this project, it is planned to organize an international Round-Robin on MCVN testing that will involve highly qualified and experienced laboratories in the U.S. and overseas. Results will be analyzed in accordance with ASTM E691-11 [11] in order to establish certified values of absorbed energy and maximum force at low, high, and super-high energy levels.

\section{Acknowledgements}

I express my gratitude to Jean-Louis Puzzolante and Marc Scibetta, my former colleagues at SCK $\cdot \mathrm{CEN}$ in Belgium, for providing me the KLST specimens of JRQ steel. I also thank Chris McCowan and Ray Santoyo (NIST) for their support during the preliminary phase of this project. 


\section{Bibliography}

[1] D. E. Driscoll, "Reproducibility of Charpy Impact Test, " in Symposium on Impact Testing, ASTM STP 176 , F. G. Tatnall, cd., American Society for Testing and Materials, Philadelphia, PA, 1955, pp. 70-75.

[2] T. A. Sicwert, M. P. Manahan, C. N. McCowan, J. M. Holt, F. J. Marsh, and E. A. Ruth, "The History and Importance of Impact Testing, " in Pendulmm Impact Testing: A Centmry of Progress, ASTM STP 1380, T. A. Siewert and M. P. Manahan, Sr., Eds., American Socicty for Testing and Materials, West Conshohocken, PA, 1999, pp. 3-16.

[3] C. N. McCowan, T. A. Sicwert, and D. P. Vigliotti, "Charpy Verification Program: Reports Covering 19892002," NIST Technical Note 1500-9, September 2003.

[4] E. Lucon, R. Chaouadi, A. Fabry, J.-L. Puzzolante, and E. van Walle, "Characterization of materials propertics by use of full size and subsize Charpy tests: an overview of different correlation procedures," in Pendulım Impact Testing: A Century of Progress, ASTM STP 1380, T.A. Siewert, Ed., ASTM, Philadelphia, PA, 2000, pp. 146-163.

[5] ISO 14556:2000, "Steel -- Charpy V-noteh pendulum impact test -- Instrumented test method," International Organization for Standardization, www.iso.org.

[6] M. P. Manahan and C. Charles, "A Generalized Mcthodology for Obtaining Quantitative Charpy Data From Test Specimens of Nonstandard Dimensions," Nuclear Technology, Vol. 90, 1990, pp. 245-259.

[7] ASTM E2248-09, "Standard Test Method for Impact Testing of Miniaturized Charpy V-Notch Specimens," ASTM Book of Standards, Vol. 03.01.

[8] Home page of the Generation IV lnternational Forum, http:/www.gen-4.org/.

[9] IFMIF home page, http://www.frascati.cnca.it/ifmif/.

[10] M. P. Manahan, F. J. Martin, and R. B. Stonesifcr, "Results of the ASTM Instrumented/Miniaturized Round Robin Test Program", in Pendulım Impact Testing: A Century of Progress, ASTM STP 1380, T. A. Siewert and M. P. Manahan, Sr., Eds., American Society for Testing and Materials, West Conshohocken, PA, 1999, pp. 223-241.

[11] ASTM E691-11, "Standard Practice for Conducting an Interlaboratory Study to Determine the Precision of a Test Method," ASTM Book of Standards, Vol. 14.02.

[12] E. Lucon, G. Roebben, J.-L. Puzzolante, and A. Lamberty, "Impact Characterization of Sub-Size Charpy VNotch Specimens Prepared from Full-Sizc Certified Reference Charpy V-Notch Test Picces," Journal of ASTM International, Vol. 2, No. 7, 2005.

[13] E. Lucon, "Europcan activity on instrumented impact testing of subsize Charpy V-notch specimens (ESIS TC5)," in Pendulum Impact Testing: A Century of Progress, ASTM STP 1380. T. A. Siewert and M. P. Manahan, Sr., Eds., Ameriean Society for Testing and Materials, West Conshohocken, PA, 1999, pp. $242-$ 252.

[14] "Reference manual on the IAEA JRQ correlation monitor" steel for irradiation damage studies," IAEATECDOC-1230, International Atomic Energy Authority, Vienna (Austria) July 2001.

[15] ASTM E2298-09, "Standard Test Method for Instrumented Impact Testing of Metallic Materials," ASTM Book of Standards, Vol. 03.01.

[16] C. N. McCowan, J. D. Splett, and E. Lucon, "Dynamic Force Measurement: Instrumented Charpy Impact Tcsting," NISTIR 6652, National Institute of Standards and Technology, January 2008.

[17] C. N. McCowan, E. Lucon, and R. L. Santoyo, "Evaluation of Bias for Two Charpy Impact Machines with the Same Instrumented Striker," Jounnal of ASTM International, Vol. 8, No. 5, 2011.

[18] C. N. McCowan, T. A. Sicwert, and D. P. Vigliotti, "The NIST Charpy V-notch Verification Program: Ovcrview and Opcrating Procedures," in Charpy Verification Program: Reports Covering 1989-2002, NIST Technical Note 1500-9, September 2003.

[19] F. Grubbs, "Procedures for Detecting Outlying Obscrvations in Samples," Technometrics, 11(1), 1969, pp. $1-21$.

[20] E. Lucon, R. Chaouadi, and E. van Wallc, "Different Approaches for the Verification of Force Values Measured with Instrumented Charpy Strikers," in Pendmlmm Impact Machines: Procedmes and Specimens, 
ASTM STP 1476 (Eds: T. Siewert, M. Manahan, and C. McCowan), ASTM, West Conshohocken, PA, 2006, pp. 95-102.

[21] E. Lucon, "On the Effectiveness of the Dynamic Force Adjustment for Reducing the Scatter of Instrumented Charpy Results," Journal of ASTM International, Vol. 6, No. 1 (January 2009).

[22] M. A. Sokolov and D. J. Alexander, "An Improved Correlation Procedure for Subsize and Full-Size Charpy Impact Specimen Data, ” NUREG/CR-6379, ORNL-6888, March 1997.

[23] E. Lucon, R. Chaouadi, A. Fabry, J.-L. Puzzolante, and E. van Walle, "Characterisation of materials properties by use of full size and subsize Charpy tests: an overview of different correlation procedures," in Pendulum Impact Testing: A Century of Progress, ASTM STP 1380 (Ed: T.A. Siewert), ASTM, Philadelphia, PA 2000, pp. 146-163.

[24] W. R. Corwin, R. L. Klueh, and J. M. Vitek, "Effect of specimen size and nickel content on the impact properties of 12 Cr-1 MoVW ferritic steel," Journal of Nuclear Materials Vol. 122, Issues 1-3, 1984, pp. 343-348.

[25] W. R. Corwin, and A. M. Hougland, "Effect of Specimen Size and Material Condition on the Charpy Impact Properties of 9Cr-1Mo-V-Nb Steel," in The Use of Small-Scale Specimens for Testing Irradiated Material, ASTM STP 888 (Eds: W. R. Corwin, G. E. Lucas), ASTM, Philadelphia, PA 1986, pp. 325-338.

[26] G. E. Lucas, G. R. Odette, J. W. Sheckherd, P. McConnell, and J. Perrin, "Subsized Bend and Charpy V-Notch Specimens for Irradiated Testing," in The Use of Small-Scale Specimens for Testing Irradiated Material, ASTM STP 888 (Eds: W. R. Corwin, G. E. Lucas), ASTM, Philadelphia, PA 1986, pp. 304-324.

[27] G. E. Lucas, G. R. Odette, J. W. Sheckherd, and M. R. Krishnadev, "Recent Progress in Subsized Charpy Impact Specimen Testing for Fusion Reactor Materials Development," Fusion Technology Vol. 10, 1986, pp. 728-733.

[28] B. S. Louden, A. S. Kumar, F. A. Garner, M. L. Hamilton, and W. L. Hu, "The influence of specimen size on charpy impact testing of unirradiated HT-9," Journal of Nuclear Materials Vol. 155-157, 1988, pp. $662-667$.

[29] C. N. McCowan, J. Pauwels, G. Revise, and H. Nakano, "International Comparison of Impact Verification Programs," in Pendulum Impact Testing: A Century of Progress, ASTM STP 1380, T.A. Siewert, ed., ASTM, Philadelphia, PA, 2000, pp. 73-89.

[30] C. N. McCowan and D. P. Vigliotti, "The Influence of Shear Lip Symmetry on the Absorbed Energy of Charpy Impact Specimens," in Charpy Verification Program: Reports Covering 1989-2002, NIST Technical Note 1500-9, September 2003.

[31] Student (pseudonym of W. S. Gossett), "The Probable Error of a Mean," Biometrika (1908), Vol. 6(1), pp. 1-25.

[32] R. G. Chandavale and T. Dutta, "Correction of Charpy Impact Values for Kinetic Energy of Test Specimens," in Pendulum Impact Machines: Procedures and Specimens for Verification, ASTM STP 1248, T.A. Siewert and A, K. Schmieder, eds., ASTM, Philadelphia, PA, 1995, pp. 221-231. 





